\title{
Normalized Cuts and Image Segmentation
}

\author{
Jianbo Shi and Jitendra Malik, Member, IEEE
}

\begin{abstract}
We propose a novel approach for solving the perceptual grouping problem in vision. Rather than focusing on local features and their consistencies in the image data, our approach aims at extracting the global impression of an image. We treat image segmentation as a graph partitioning problem and propose a novel global criterion, the normalized cut, for segmenting the graph. The normalized cut criterion measures both the total dissimilarity between the different groups as well as the total similarity within the groups. We show that an efficient computational technique based on a generalized eigenvalue problem can be used to optimize this criterion. We have applied this approach to segmenting static images, as well as motion sequences, and found the results to be very encouraging.
\end{abstract}

Index Terms-Grouping, image segmentation, graph partitioning.

\section{INTRODUCTION}

$\mathrm{N}$ EARLY 75 years ago, Wertheimer [24] pointed out the importance of perceptual grouping and organization in vision and listed several key factors, such as similarity, proximity, and good continuation, which lead to visual grouping. However, even to this day, many of the computational issues of perceptual grouping have remained unresolved. In this paper, we present a general framework for this problem, focusing specifically on the case of image segmentation.

Since there are many possible partitions of the domain $I$ of an image into subsets, how do we pick the "right" one? There are two aspects to be considered here. The first is that there may not be a single correct answer. A Bayesian view is appropriate-there are several possible interpretations in the context of prior world knowledge. The difficulty, of course, is in specifying the prior world knowledge. Some of it is low level, such as coherence of brightness, color, texture, or motion, but equally important is mid- or highlevel knowledge about symmetries of objects or object models. The second aspect is that the partitioning is inherently hierarchical. Therefore, it is more appropriate to think of returning a tree structure corresponding to a hierarchical partition instead of a single "flat" partition.

This suggests that image segmentation based on lowlevel cues cannot and should not aim to produce a complete final "correct" segmentation. The objective should instead be to use the low-level coherence of brightness, color, texture, or motion attributes to sequentially come up with hierarchical partitions. Mid- and high-level knowledge can be used to either confirm these groups or select some for further attention. This attention could result in further repartitioning or grouping. The key point is that image partitioning is

- J. Shi is with the Robotics Institute, Carnegie Mellon University, 5000 Forbes Ave., Pittsburgh, PA 15213. E-mail: jshi@cs.cmu.edu

- J. Malik is with the Electrical Engineering and Computer Science Division, University of California at Berkeley, Berkeley, CA 94720.

E-mail:malik@cs.berkeley.edu.

Manuscript received 4 Feb. 1998; accepted 16 Nov. 1999.

Recommended for acceptance by M. Shah.

For information on obtaining reprints of this article, please send e-mail to: tpami@computer.org, and reference IEEECS Log Number 107618. to be done from the big picture downward, rather like a painter first marking out the major areas and then filling in the details.

Prior literature on the related problems of clustering, grouping and image segmentation is huge. The clustering community [12] has offered us agglomerative and divisive algorithms; in image segmentation, we have region-based merge and split algorithms. The hierarchical divisive approach that we advocate produces a tree, the dendrogram. While most of these ideas go back to the 1970s (and earlier), the 1980s brought in the use of Markov Random Fields [10] and variational formulations [17], [2], [14]. The MRF and variational formulations also exposed two basic questions:

1. What is the criterion that one wants to optimize?

2. Is there an efficient algorithm for carrying out the optimization?

Many an attractive criterion has been doomed by the inability to find an effective algorithm to find its minimum-greedy or gradient descent type approaches fail to find global optima for these high-dimensional, nonlinear problems.

Our approach is most related to the graph theoretic formulation of grouping. The set of points in an arbitrary feature space are represented as a weighted undirected graph $G=(V, E)$, where the nodes of the graph are the points in the feature space, and an edge is formed between every pair of nodes. The weight on each edge, $w(\boldsymbol{i}, \boldsymbol{j})$, is a function of the similarity between nodes $i$ and $j$.

In grouping, we seek to partition the set of vertices into disjoint sets $\mathbf{V}_{1}, \mathbf{V}_{2}, \ldots, \mathbf{V}_{m}$, where by some measure the similarity among the vertices in a set $\mathbf{V}_{i}$ is high and, across different sets $\mathbf{V}_{i}, \mathbf{V}_{j}$ is low.

To partition a graph, we need to also ask the following questions:

1. What is the precise criterion for a good partition?

2. How can such a partition be computed efficiently?

In the image segmentation and data clustering community, there has been much previous work using variations of the minimal spanning tree or limited neighborhood set approaches. Although those use efficient computational 
methods, the segmentation criteria used in most of them are based on local properties of the graph. Because perceptual grouping is about extracting the global impressions of a scene, as we saw earlier, this partitioning criterion often falls short of this main goal.

In this paper, we propose a new graph-theoretic criterion for measuring the goodness of an image partition-the normalized cut. We introduce and justify this criterion in Section 2. The minimization of this criterion can be formulated as a generalized eigenvalue problem. The eigenvectors can be used to construct good partitions of the image and the process can be continued recursively as desired (Section 2.1). Section 3 gives a detailed explanation of the steps of our grouping algorithm. In Section 4, we show experimental results. The formulation and minimization of the normalized cut criterion draws on a body of results from the field of spectral graph theory (Section 5). Relationship to work in computer vision is discussed in Section 6 and comparison with related eigenvector based segmentation methods is represented in Section 6.1. We conclude in Section 7.

The main results in this paper were first presented in [20].

\section{Grouping as Graph Partitioning}

A graph $\mathbf{G}=(\mathbf{V}, \mathbf{E})$ can be partitioned into two disjoint sets, $A, B, A \cup B=V, A \cap B=\emptyset$, by simply removing edges connecting the two parts. The degree of dissimilarity between these two pieces can be computed as total weight of the edges that have been removed. In graph theoretic language, it is called the cut:

$$
\operatorname{cut}(A, B)=\sum_{u \in A, v \in B} w(u, v) .
$$

The optimal bipartitioning of a graph is the one that minimizes this cut value. Although there are an exponential number of such partitions, finding the minimum cut of a graph is a well-studied problem and there exist efficient algorithms for solving it.

$\mathrm{Wu}$ and Leahy [25] proposed a clustering method based on this minimum cut criterion. In particular, they seek to partition a graph into k-subgraphs such that the maximum cut across the subgroups is minimized. This problem can be efficiently solved by recursively finding the minimum cuts that bisect the existing segments. As shown in $\mathrm{Wu}$ and Leahy's work, this globally optimal criterion can be used to produce good segmentation on some of the images.

However, as $\mathrm{Wu}$ and Leahy also noticed in their work, the minimum cut criteria favors cutting small sets of isolated nodes in the graph. This is not surprising since the cut defined in (1) increases with the number of edges going across the two partitioned parts. Fig. 1 illustrates one such case. Assuming the edge weights are inversely proportional to the distance between the two nodes, we see the cut that partitions out node $n_{1}$ or $n_{2}$ will have a very small value. In fact, any cut that partitions out individual nodes on the right half will have smaller cut value than the cut that partitions the nodes into the left and right halves.

To avoid this unnatural bias for partitioning out small sets of points, we propose a new measure of disassociation

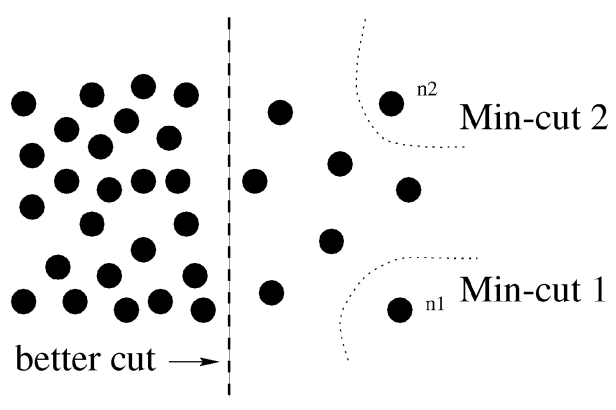

Fig. 1. A case where minimum cut gives a bad partition.

between two groups. Instead of looking at the value of total edge weight connecting the two partitions, our measure computes the cut cost as a fraction of the total edge connections to all the nodes in the graph. We call this disassociation measure the normalized cut (Ncut):

$$
N \operatorname{cut}(A, B)=\frac{\operatorname{cut}(A, B)}{\operatorname{assoc}(A, V)}+\frac{\operatorname{cut}(A, B)}{\operatorname{assoc}(B, V)},
$$

where $\operatorname{assoc}(A, V)=\sum_{u \in A, t \in V} w(u, t)$ is the total connection from nodes in A to all nodes in the graph and $\operatorname{assoc}(B, V)$ is similarly defined. With this definition of the disassociation between the groups, the cut that partitions out small isolated points will no longer have small Ncut value, since the cut value will almost certainly be a large percentage of the total connection from that small set to all other nodes. In the case illustrated in Fig. 1, we see that the cut $_{1}$ value across node $n_{1}$ will be 100 percent of the total connection from that node.

In the same spirit, we can define a measure for total normalized association within groups for a given partition:

$$
\operatorname{Nassoc}(A, B)=\frac{\operatorname{assoc}(A, A)}{\operatorname{assoc}(A, V)}+\frac{\operatorname{assoc}(B, B)}{\operatorname{assoc}(B, V)},
$$

where $\operatorname{assoc}(A, A)$ and $\operatorname{assoc}(B, B)$ are total weights of edges connecting nodes within $A$ and $B$, respectively. We see again this is an unbiased measure, which reflects how tightly on average nodes within the group are connected to each other.

Another important property of this definition of association and disassociation of a partition is that they are naturally related:

$$
\begin{aligned}
N \operatorname{cut}(A, B)= & \frac{\operatorname{cut}(A, B)}{\operatorname{assoc}(A, V)}+\frac{\operatorname{cut}(A, B)}{\operatorname{assoc}(B, V)} \\
= & \frac{\operatorname{assoc}(A, V)-\operatorname{assoc}(A, A)}{\operatorname{assoc}(A, V)} \\
& \quad+\frac{\operatorname{assoc}(B, V)-\operatorname{assoc}(B, B)}{\operatorname{assoc}(B, V)} \\
= & 2-\left(\frac{\operatorname{assoc}(A, A)}{\operatorname{assoc}(A, V)}+\frac{\operatorname{assoc}(B, B)}{\operatorname{assoc}(B, V)}\right) \\
= & 2-N \operatorname{assoc}(A, B) .
\end{aligned}
$$

Hence, the two partition criteria that we seek in our grouping algorithm, minimizing the disassociation between the groups and maximizing the association within the 
groups, are in fact identical and can be satisfied simultaneously. In our algorithm, we will use this normalized cut as the partition criterion.

Unfortunately, minimizing normalized cut exactly is NPcomplete, even for the special case of graphs on grids. The proof, due to Papadimitriou, can be found in Appendix A. However, we will show that, when we embed the normalized cut problem in the real value domain, an approximate discrete solution can be found efficiently.

\subsection{Computing the Optimal Partition}

Given a partition of nodes of a graph, V, into two sets $\mathrm{A}$ and B, let $x$ be an $N=|V|$ dimensional indicator vector, $x_{i}=1$ if node $i$ is in A and -1 , otherwise. Let $d(i)=\sum_{j} w(i, j)$ be the total connection from node $i$ to all other nodes. With the definitions $x$ and $d$, we can rewrite $\operatorname{Ncut}(A, B)$ as:

$$
\begin{aligned}
N \operatorname{cut}(A, B)= & \frac{\operatorname{cut}(A, B)}{\operatorname{assoc}(A, V)}+\frac{\operatorname{cut}(B, A)}{\operatorname{assoc}(B, V)} \\
= & \frac{\sum_{\left(\boldsymbol{x}_{i}>0, \boldsymbol{x}_{j}<0\right)}-w_{i j} \boldsymbol{x}_{i} \boldsymbol{x}_{j}}{\sum_{\boldsymbol{x}_{i}>0} \boldsymbol{d}_{i}} \\
& +\frac{\sum_{\left(\boldsymbol{x}_{i}<0, \boldsymbol{x}_{j}>0\right)}-w_{i j} \boldsymbol{x}_{i} \boldsymbol{x}_{j}}{\sum_{\boldsymbol{x}_{i}<0} \boldsymbol{d}_{i}} .
\end{aligned}
$$

Let $\mathbf{D}$ be an $N \times N$ diagonal matrix with $d$ on its diagonal, W be an $N \times N$ symmetrical matrix with $\mathrm{W}(\mathrm{i}, \mathrm{j})=w_{i j}$,

$$
k=\frac{\sum_{x_{i}>0} \boldsymbol{d}_{i}}{\sum_{i} \boldsymbol{d}_{i}},
$$

and 1 be an $N \times 1$ vector of all ones. Using the fact $\frac{1+x}{2}$ and $\frac{1-x}{2}$ are indicator vectors for $x_{i}>0$ and $x_{i}<0$, respectively, we can rewrite $4[N \operatorname{cut}(\boldsymbol{x})]$ as:

$$
\begin{aligned}
& =\frac{(\mathbf{1}+x)^{T}(\mathbf{D}-\mathbf{W})(\mathbf{1}+x)}{k \mathbf{1}^{T} \mathbf{D} \mathbf{1}}+\frac{(\mathbf{1}-x)^{T}(\mathbf{D}-\mathbf{W})(\mathbf{1}-\boldsymbol{x})}{(1-k) \mathbf{1}^{T} \mathbf{D} \mathbf{1}} \\
& =\frac{\left(x^{T}(\mathbf{D}-\mathbf{W}) \boldsymbol{x}+\mathbf{1}^{T}(\mathbf{D}-\mathbf{W}) \mathbf{1}\right)}{k(1-k) \mathbf{1}^{T} \mathbf{D} \mathbf{1}}+\frac{2(1-2 k) \mathbf{1}^{T}(\mathbf{D}-\mathbf{W}) \boldsymbol{x}}{k(1-k) \mathbf{1}^{T} \mathbf{D} \mathbf{1}} .
\end{aligned}
$$

Let

$$
\begin{aligned}
\alpha(x) & =x^{T}(\mathbf{D}-\mathbf{W}) \boldsymbol{x}, \\
\beta(x) & =\mathbf{1}^{T}(\mathbf{D}-\mathbf{W}) \boldsymbol{x}, \\
\gamma & =\mathbf{1}^{T}(\mathbf{D}-\mathbf{W}) \mathbf{1},
\end{aligned}
$$

and

$$
M=\mathbf{1}^{T} \mathbf{D} \mathbf{1},
$$

we can then further expand the above equation as:

$$
\begin{aligned}
& =\frac{(\alpha(\boldsymbol{x})+\gamma)+2(1-2 k) \beta(x)}{k(1-k) M} \\
& =\frac{(\alpha(x)+\gamma)+2(1-2 k) \beta(x)}{k(1-k) M}-\frac{2(\alpha(x)+\gamma)}{M}+\frac{2 \alpha(x)}{M}+\frac{2 \gamma}{M} .
\end{aligned}
$$

Dropping the last constant term, which in this case equals 0 , we get

$$
\begin{aligned}
= & \frac{\left(1-2 k+2 k^{2}\right)(\alpha(x)+\gamma)+2(1-2 k) \beta(x)}{k(1-k) M}+\frac{2 \alpha(x)}{M} \\
= & \frac{\frac{\left(1-2 k+2 k^{2}\right)}{(1-k)^{2}}(\alpha(x)+\gamma)+\frac{2(1-2 k)}{(1-k)^{2}} \beta(x)}{\frac{k}{1-k} M} \\
& +\frac{2 \alpha(x)}{M} .
\end{aligned}
$$

Letting $b=\frac{k}{1-k}$, and since $\gamma=0$, it becomes

$$
\begin{aligned}
= & \frac{\left(1+b^{2}\right)(\alpha(\boldsymbol{x})+\gamma)+2\left(1-b^{2}\right) \beta(\boldsymbol{x})}{b M}+\frac{2 b \alpha(\boldsymbol{x})}{b M} \\
= & \frac{\left(1+b^{2}\right)(\alpha(\boldsymbol{x})+\gamma)}{b M}+\frac{2\left(1-b^{2}\right) \beta(\boldsymbol{x})}{b M}+\frac{2 b \alpha(\boldsymbol{x})}{b M}-\frac{2 b \gamma}{b M} \\
= & \frac{\left(1+b^{2}\right)\left(\boldsymbol{x}^{T}(\mathbf{D}-\mathbf{W}) \boldsymbol{x}+\mathbf{1}^{T}(\mathbf{D}-\mathbf{W}) \mathbf{1}\right)}{b \mathbf{1}^{T} \mathbf{D} \mathbf{1}} \\
& +\frac{2\left(1-b^{2}\right) \mathbf{1}^{T}(\mathbf{D}-\mathbf{W}) \boldsymbol{x}}{b \mathbf{1}^{T} \mathbf{D} \mathbf{1}} \\
& +\frac{2 b \boldsymbol{x}^{T}(\mathbf{D}-\mathbf{W}) \boldsymbol{x}}{b \mathbf{1}^{T} \mathbf{D} \mathbf{1}}-\frac{2 b \mathbf{1}^{T}(\mathbf{D}-\mathbf{W}) \mathbf{1}}{b \mathbf{1}^{T} \mathbf{D} \mathbf{1}} \\
= & \frac{(\mathbf{1}+\boldsymbol{x})^{T}(\mathbf{D}-\mathbf{W})(\mathbf{1}+\boldsymbol{x})}{b \mathbf{1}^{T} \mathbf{D} \mathbf{1}} \\
& +\frac{b^{2}(\mathbf{1}-\boldsymbol{x})^{T}(\mathbf{D}-\mathbf{W})(\mathbf{1}-\boldsymbol{x})}{b \mathbf{1}^{T} \mathbf{D} \mathbf{1}} \\
& \quad-\frac{2 b(\mathbf{1}-\boldsymbol{x})^{T}(\mathbf{D}-\mathbf{W})(\mathbf{1}+\boldsymbol{x})}{b \mathbf{1}^{T} \mathbf{D} \mathbf{1}} \\
= & \frac{[(\mathbf{1}+\boldsymbol{x})-b(\mathbf{1}-\boldsymbol{x})]^{T}(\mathbf{D}-\mathbf{W})[(\mathbf{1}+\boldsymbol{x})-b(\mathbf{1}-\boldsymbol{x})]}{b \mathbf{1}^{T} \mathbf{D} \mathbf{1}} .
\end{aligned}
$$

Setting $y=(\mathbf{1}+x)-b(\mathbf{1}-\boldsymbol{x})$, it is easy to see that

$$
\boldsymbol{y}^{T} \mathbf{D} \mathbf{1}=\sum_{x_{i}>0} \boldsymbol{d}_{i}-b \sum_{x_{i}<0} \boldsymbol{d}_{i}=0
$$

since $b=\frac{k}{1-k}=\frac{\sum_{x_{i}>0} d_{i}}{\sum_{x_{i}<0} d_{i}}$ and

$$
\begin{aligned}
\boldsymbol{y}^{T} \mathbf{D} \boldsymbol{y} & =\sum_{x_{i}>0} \boldsymbol{d}_{i}+b^{2} \sum_{x_{i}<0} \boldsymbol{d}_{i} \\
& =b \sum_{x_{i}<0} \boldsymbol{d}_{i}+b^{2} \sum_{x_{i}<0} \boldsymbol{d}_{i} \\
& =b\left(\sum_{x_{i}<0} \boldsymbol{d}_{i}+b \sum_{x_{i}<0} \boldsymbol{d}_{i}\right) \\
& =b \mathbf{1}^{T} \mathbf{D} \mathbf{1} .
\end{aligned}
$$

Putting everything together we have,

$$
\min _{\boldsymbol{x}} N \operatorname{cut}(\boldsymbol{x})=\min _{\boldsymbol{y}} \frac{\boldsymbol{y}^{T}(\mathbf{D}-\mathbf{W}) \boldsymbol{y}}{\boldsymbol{y}^{T} \mathbf{D} \boldsymbol{y}},
$$

with the condition $\boldsymbol{y}(i) \in\{1,-b\}$ and $y^{T} \mathbf{D} \mathbf{1}=0$.

Note that the above expression is the Rayleigh quotient [11]. If $y$ is relaxed to take on real values, we can minimize (5) by solving the generalized eigenvalue system,

$$
(\mathbf{D}-\mathbf{W}) \boldsymbol{y}=\lambda \mathbf{D} y .
$$

However, we have two constraints on $y$ which come from the condition on the corresponding indicator vector $x$. First, consider the constraint $\boldsymbol{y}^{T} \mathbf{D} \mathbf{1}=0$. We can show this constraint on $y$ is automatically satisfied by the solution of the generalized eigensystem. We will do so by first 
transforming (6) into a standard eigensystem and showing the corresponding condition is satisfied there. Rewrite (6) as

$$
\mathbf{D}^{-\frac{1}{2}}(\mathbf{D}-\mathbf{W}) \mathbf{D}^{-\frac{1}{2}} z=\lambda z
$$

where $z=\mathbf{D}^{\frac{1}{2}} \boldsymbol{y}$. One can easily verify that $z_{0}=\mathbf{D}^{\frac{1}{2}} \mathbf{1}$ is an eigenvector of (7) with eigenvalue of 0 . Furthermore, $\mathbf{D}^{-\frac{1}{2}}(\mathbf{D}-\mathbf{W}) \mathbf{D}^{-\frac{1}{2}}$ is symmetric positive semidefinite since $(\mathbf{D}-\mathbf{W})$, also called the Laplacian matrix, is known to be positive semidefinite [18]. Hence, $z_{0}$ is, in fact, the smallest eigenvector of (7) and all eigenvectors of (7) are perpendicular to each other. In particular, $z_{1}$, the second smallest eigenvector, is perpendicular to $z_{0}$. Translating this statement back into the general eigensystem (6), we have: 1) $y_{0}=1$ is the smallest eigenvector with eigenvalue of 0 and 2) $0=z_{1}^{T} z_{0}=\boldsymbol{y}_{1}^{T} \mathbf{D} \mathbf{1}$, where $\boldsymbol{y}_{1}$ is the second smallest eigenvector of (6).

Now, recall a simple fact about the Rayleigh quotient [11]:

Let $\mathbf{A}$ be a real symmetric matrix. Under the constraint that $x$ is orthogonal to the $\mathrm{j}-1$ smallest eigenvectors $x_{1}, \ldots, x_{j-1}$, the quotient $\frac{x^{T} \mathbf{A} x}{x^{T} x}$ is minimized by the next smallest eigenvector $x_{j}$ and its minimum value is the corresponding eigenvalue $\lambda_{j}$.

As a result, we obtain:

$$
z_{1}=\arg \cdot \min _{z^{T} z_{0}=0} \frac{z^{T} \mathbf{D}^{-\frac{1}{2}}(\mathbf{D}-\mathbf{W}) \mathbf{D}^{-\frac{1}{2}} z}{z^{T} z}
$$

and, consequently,

$$
\boldsymbol{y}_{1}=\arg _{\min _{\boldsymbol{y}^{T} \mathbf{D} \mathbf{1}=0}} \frac{\boldsymbol{y}^{T}(\mathbf{D}-\mathbf{W}) \boldsymbol{y}}{\boldsymbol{y}^{T} \mathbf{D} \boldsymbol{y}} .
$$

Thus, the second smallest eigenvector of the generalized eigensystem (6) is the real valued solution to our normalized cut problem. The only reason that it is not necessarily the solution to our original problem is that the second constraint on $y$ that $y(i)$ takes on two discrete values is not automatically satisfied. In fact, relaxing this constraint is what makes this optimization problem tractable in the first place. We will show in Section 3 how this real valued solution can be transformed into a discrete form.

A similar argument can also be made to show that the eigenvector with the third smallest eigenvalue is the real valued solution that optimally subpartitions the first two parts. In fact, this line of argument can be extended to show that one can subdivide the existing graphs, each time using the eigenvector with the next smallest eigenvalue. However, in practice, because the approximation error from the real valued solution to the discrete valued solution accumulates with every eigenvector taken and all eigenvectors have to satisfy a global mutual orthogonality constraint, solutions based on higher eigenvectors become unreliable. It is best to restart solving the partitioning problem on each subgraph individually.

It is interesting to note that, while the second smallest eigenvector $y$ of (6) only approximates the optimal normalized cut solution, it exactly minimizes the following problem:

$$
\inf _{\boldsymbol{y}^{T} \mathbf{D} \mathbf{1}=0} \frac{\sum_{i} \sum_{j}(\boldsymbol{y}(i)-\boldsymbol{y}(j))^{2} w_{i j}}{\sum_{i} \boldsymbol{y}(i)^{2} \mathbf{d}(i)},
$$

in real-valued domain, where $\mathbf{d}(i)=\mathbf{D}(i, i)$. Roughly speaking, this forces the indicator vector $y$ to take similar values for nodes $i$ and $j$ that are tightly coupled (large $w_{i j}$ ).

In summary, we propose using the normalized cut criterion for graph partitioning and we have shown how this criterion can be computed efficiently by solving a generalized eigenvalue problem.

\section{The Grouping Algorithm}

Our grouping algorithm consists of the following steps:

1. Given an image or image sequence, set up a weighted graph $\mathbf{G}=(\mathbf{V}, \mathbf{E})$ and set the weight on the edge connecting two nodes to be a measure of the similarity between the two nodes.

2. Solve $(\mathbf{D}-\mathbf{W}) x=\lambda \mathbf{D} x$ for eigenvectors with the smallest eigenvalues.

3. Use the eigenvector with the second smallest eigenvalue to bipartition the graph.

4. Decide if the current partition should be subdivided and recursively repartition the segmented parts if necessary.

The grouping algorithm, as well as its computational complexity, can be best illustrated by using the following example.

\subsection{Example: Brightness Images}

Fig. 2 shows an image that we would like to segment. The steps are:

1. Construct a weighted graph $\mathbf{G}=(\mathbf{V}, \mathbf{E})$ by taking each pixel as a node and connecting each pair of pixels by an edge. The weight on that edge should reflect the likelihood that the two pixels belong to one object. Using just the brightness value of the pixels and their spatial location, we can define the graph edge weight connecting the two nodes $i$ and $j$ as:

$$
\begin{aligned}
& w_{i j}=e^{\frac{-\left\|\boldsymbol{F}_{(i)}-\boldsymbol{F}_{(j)}\right\|_{2}^{2}}{\sigma_{I}^{2}}} * \\
& \begin{cases}e^{\frac{-\left\|\boldsymbol{X}_{(i)}-\boldsymbol{X}_{(j)}\right\|_{2}^{2}}{\sigma_{X}^{2}}} & \text { if }\|X(i)-X(j)\|_{2}<r \\
0 & \text { otherwise. }\end{cases}
\end{aligned}
$$

2. Solve for the eigenvectors with the smallest eigenvalues of the system

$$
(\mathbf{D}-\mathbf{W}) \boldsymbol{y}=\lambda \mathbf{D} y .
$$

As we saw above, the generalized eigensystem in (12) can be transformed into a standard eigenvalue problem of

$$
\mathbf{D}^{-\frac{1}{2}}(\mathbf{D}-\mathbf{W}) \mathbf{D}^{-\frac{1}{2}} \boldsymbol{x}=\lambda \boldsymbol{x} .
$$

Solving a standard eigenvalue problem for all eigenvectors takes $O\left(n^{3}\right)$ operations, where $n$ is the number of nodes in the graph. This becomes impractical for image segmentation applications where $n$ is the number of pixels in an image. 


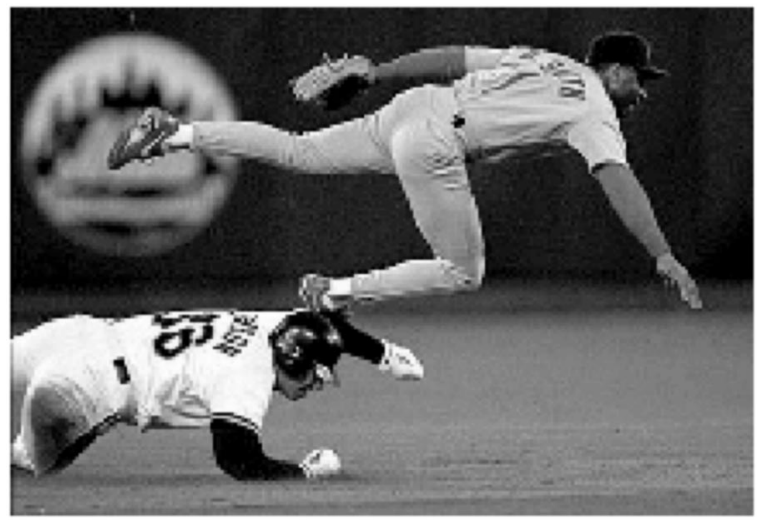

Fig. 2. A gray level image of a baseball game.

Fortunately, our graph partitioning has the following properties: 1) The graphs are often only locally connected and the resulting eigensystems are very sparse, 2) only the top few eigenvectors are needed for graph partitioning, and 3) the precision requirement for the eigenvectors is low, often only the right sign bit is required. These special properties of our problem can be fully exploited by an eigensolver called the Lanczos method. The running time of a Lanczos algorithm is $O(m n)+O(m M(n))$ [11], where $m$ is the maximum number of matrix-vector computations required and $M(n)$ is the cost of a matrix-vector computation of $\mathbf{A} x$, where $\mathbf{A}=\mathbf{D}^{-\frac{1}{2}}(\mathbf{D}-\mathbf{W}) \mathbf{D}^{-\frac{1}{2}}$. Note that the sparsity structure of $\mathbf{A}$ is identical to that of the weight matrix $\mathbf{W}$. Since $\mathbf{W}$ is sparse, so is $\mathbf{A}$ and the matrix-vector computation is only $O(n)$.

To see why this is the case, we will look at the cost of the inner product of one row of $A$ with a vector x. Let $\mathbf{y}_{i}=A_{i} \cdot \mathbf{x}=\sum_{j} A_{i j} \mathbf{x}_{j}$. For a fixed $i, A_{i j}$ is only nonzero if node $j$ is in a spatial neighborhood of $i$. Hence, there are only a fixed number of operations required for each $A_{i} \cdot \mathrm{x}$ and the total cost of computing $A \mathrm{x}$ is $O(n)$.

The constant factor is determined by the size of the spatial neighborhood of a node. It turns out that we can substantially cut down additional connections from each node to its neighbors by randomly selecting the connections within the neighborhood for the weighted graph. Empirically, we have found that one can remove up to 90 percent of the total connections with each of the neighborhoods when the neighborhoods are large without affecting the eigenvector solution to the system.

Putting everything together, each of the matrixvector computations cost $O(n)$ operations with a small constant factor. The number $m$ depends on many factors [11]. In our experiments on image segmentation, we observed that $m$ is typically less than $O\left(n^{\frac{1}{2}}\right)$.

Fig. 3 shows the smallest eigenvectors computed for the generalized eigensystem with the weight matrix defined above.
3. Once the eigenvectors are computed, we can partition the graph into two pieces using the second smallest eigenvector. In the ideal case, the eigenvector should only take on two discrete values and the signs of the values can tell us exactly how to partition the graph. However, our eigenvectors can take on continuous values and we need to choose a splitting point to partition it into two parts. There are many different ways of choosing such a splitting point. One can take 0 or the median value as the splitting point or one can search for the splitting point such that the resulting partition has the best $\operatorname{Ncut}(A, B)$ value. We take the latter approach in our work. Currently, the search is done by checking $l$ evenly spaced possible splitting points, and computing the best Ncut among them. In our experiments, the values in the eigenvectors are usually well separated and this method of choosing a splitting point is very reliable even with a small $l$.

4. After the graph is broken into two pieces, we can recursively run our algorithm on the two partitioned parts. Or, equivalently, we could take advantage of the special properties of the other top eigenvectors as explained in the previous section to subdivide the graph based on those eigenvectors. The recursion stops once the Ncut value exceeds certain limit.

We also impose a stability criterion on the partition. As we saw earlier, and as we see in the eigenvectors with the seventh to ninth smallest eigenvalues (Fig. 3g-h), sometimes an eigenvector can take on the shape of a continuous function, rather that the discrete indicator function that we seek. From the view of segmentation, such an eigenvector is attempting to subdivide an image region where there is no sure way of breaking it. In fact, if we are forced to partition the image based on this eigenvector, we will see there are many different splitting points which have similar Ncut values. Hence, the partition will be highly uncertain and unstable. In our current segmentation scheme, we simply choose to ignore all those eigenvectors which have smoothly varying eigenvector values. We achieve this by imposing a stability criterion which measures the degree of smoothness in the eigenvector values. The simplest measure is based on first computing the histogram of the eigenvector values and then computing the ratio between the minimum and maximum values in the bins. When the eigenvector values are continuously varying, the values in the histogram bins will stay relatively the same and the ratio will be relatively high. In our experiments, we find that simple thresholding on the ratio described above can be used to exclude unstable eigenvectors. We have set that value to be 0.06 in all our experiments.

Fig. 4 shows the final segmentation for the image shown in Fig. 2.

\subsection{Recursive Two-Way Ncut}

In summary, our grouping algorithm consists of the following steps: 


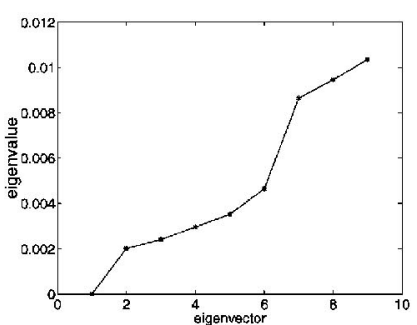

(a)

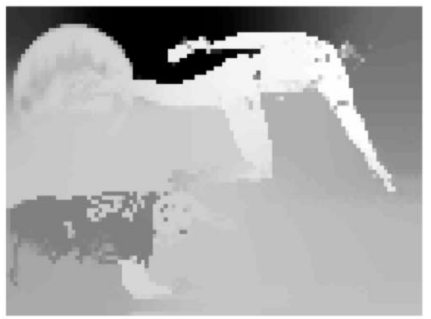

(d)

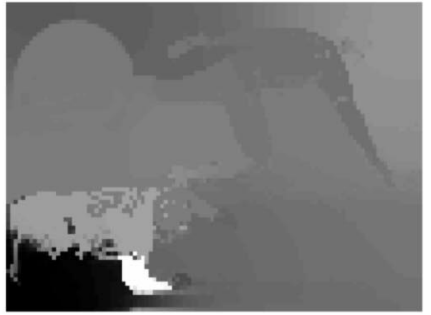

(g)

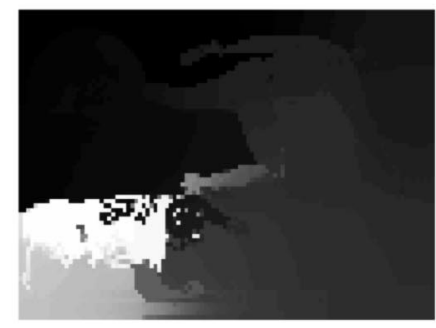

(b)

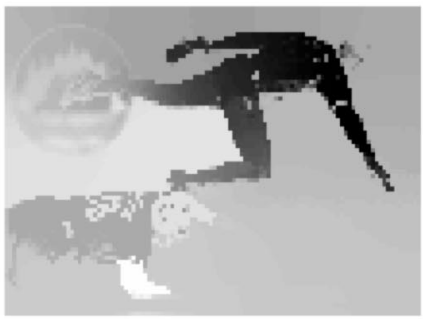

$(e)$

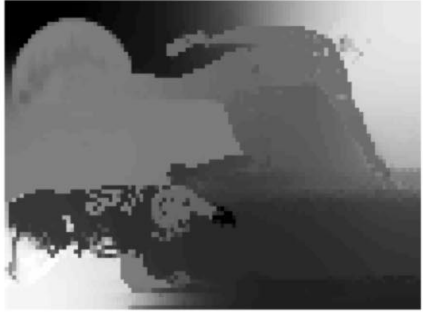

(h)

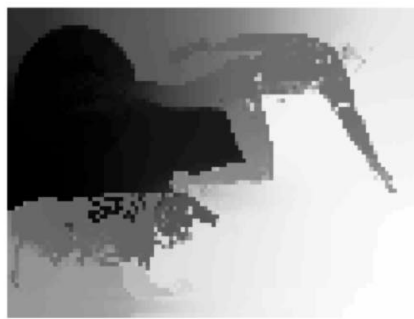

(c)

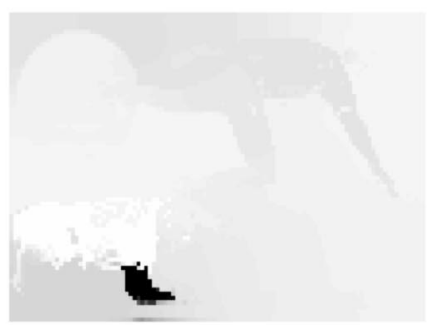

(f)

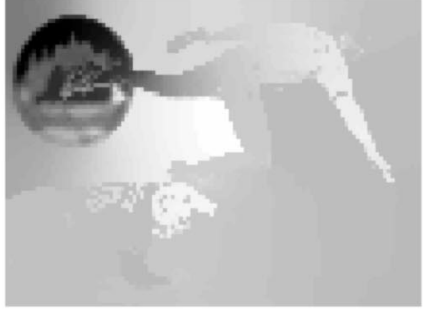

(i)

Fig. 3. Subplot (a) plots the smallest eigenvectors of the generalized eigenvalue system (11). Subplots (b)-(i) show the eigenvectors corresponding the second smallest to the ninth smallest eigenvalues of the system. The eigenvectors are reshaped to be the size of the image.

1. Given a set of features, set up a weighted graph $\mathbf{G}=(\mathbf{V}, \mathbf{E})$, compute the weight on each edge, and summarize the information into $\mathbf{W}$ and $\mathbf{D}$.

2. Solve $(\mathbf{D}-\mathbf{W}) \boldsymbol{x}=\lambda \mathbf{D} \boldsymbol{x}$ for eigenvectors with the smallest eigenvalues.

3. Use the eigenvector with the second smallest eigenvalue to bipartition the graph by finding the splitting point such that Ncut is minimized.

4. Decide if the current partition should be subdivided by checking the stability of the cut, and make sure Ncut is below the prespecified value.

5. Recursively repartition the segmented parts if necessary.

The number of groups segmented by this method is controlled directly by the maximum allowed Ncut.

\subsection{Simultanous K-Way Cut with Multiple Eigenvectors}

One drawback of the recursive 2-way cut is its treatment of the oscillatory eigenvectors. The stability criteria keeps us from cutting oscillatory eigenvectors, but it also prevents us cutting the subsequent eigenvectors which might be perfect partitioning vectors. Also, the approach is computationally wasteful; only the second eigenvector is used, whereas the next few small eigenvectors also contain useful partitioning information.

Instead of finding the partition using recursive 2-way cut as described above, one can use all of the top eigenvectors to simultanously obtain a K-way partition. In this method, the $n$ top eigenvectors are used as $n$ dimensional indicator vectors for each pixel. In the first step, a simple clustering algorithm, such as the k-means algorithm, is used to obtain an oversegmentation of the image into $k^{\prime}$ groups. No attempt is made to identify and exclude oscillatory eigenvectors - they exacerbate the oversegmentation, but that will be dealt with subsequently.

In the second step, one can proceed in the following two ways:

1. Greedy pruning: Iteratively merge two segments at a time until only $k$ segments are left. At each merge step, those two segments are merged that minimize the $k$-way $N$ cut criterion defined as:

$$
\begin{aligned}
\text { Ncut } & =\frac{\operatorname{cut}\left(\mathbf{A}_{1}, \mathbf{V}-\mathbf{A}_{1}\right)}{\operatorname{assoc}\left(\mathbf{A}_{1}, \mathbf{V}\right)}+\frac{\operatorname{cut}\left(\mathbf{A}_{2}, \mathbf{V}-\mathbf{A}_{2}\right)}{\operatorname{assoc}\left(\mathbf{A}_{2}, \mathbf{V}\right)}+\ldots \\
+ & \frac{\operatorname{cut}\left(\mathbf{A}_{k}, \mathbf{A}-\mathbf{A}_{k}\right)}{\operatorname{assoc}\left(\mathbf{A}_{k}, \mathbf{V}\right)}
\end{aligned}
$$




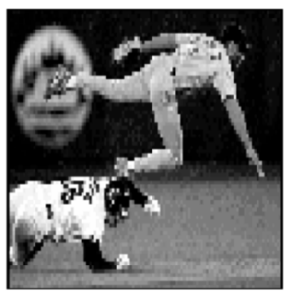

(a)

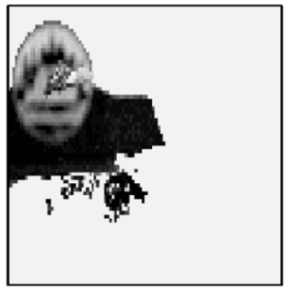

(e)

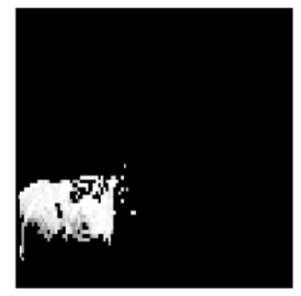

(b)

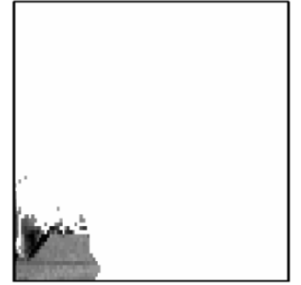

(f)

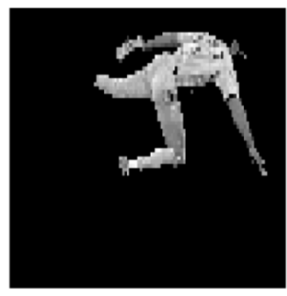

(c)

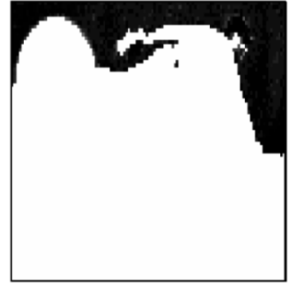

(g)

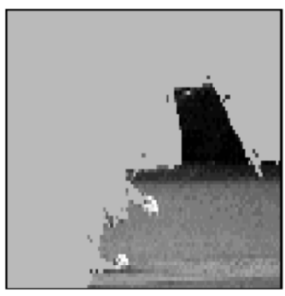

(d)

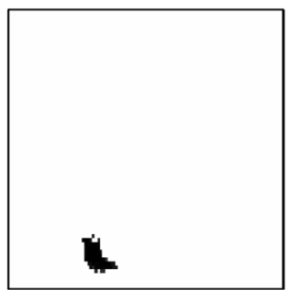

(h)

Fig. 4. (a) shows the original image of size $80 \times 100$. Image intensity is normalized to lie within 0 and 1 . Subplots (b)-(h) show the components of the partition with $N$ cut value less than 0.04. Parameter setting: $\sigma_{I}=0.1, \sigma_{X}=4.0, r=5$.

where $\mathbf{A}_{i}$ is the $i$ th subset of whole set $\mathbf{V}$.

This computation can be efficiently carried out by iteratively updating the compacted weight matrix $\mathbf{W}^{c}$, with $\mathbf{W}^{c}(i, j)=\operatorname{assoc}\left(\mathbf{A}_{i}, \mathbf{A}_{j}\right)$.

2. Global recursive cut. From the initial $k^{\prime}$ segments, we can build a condensed graph $\mathbf{G}^{c}=\left(\mathbf{V}^{c}, \mathbf{E}^{c}\right)$, where each segment $\mathbf{A}_{i}$ corresponds to a node $\mathbf{V}_{i}^{c}$ of the graph. The weight on each graph edge $\mathbf{W}^{c}(i, j)$ is defined to be $\operatorname{assoc}\left(\mathbf{A}_{i}, \mathbf{A}_{j}\right)$, the total edge weights from elements in $\mathbf{A}_{i}$ to elements in $\mathbf{A}_{j}$. From this condensed graph, we then recursively bipartition the graph according the Ncut criterion. This can be carried out either with the generalized eigenvalue system, as in Section 3.2, or with exhaustive search in the discrete domain. Exhaustive search is possible in this case since $k^{\prime}$ is small, typically $k^{\prime} \leq 100$.

We have experimented with this simultanous $k$-way cut method on our recent test images. However, the results presented in this paper are all based on the recursive 2-way partitioning algorithm outlined in Section 3.2.

\section{EXPERIMENTS}

We have applied our grouping algorithm to image segmentation based on brightness, color, texture, or motion information. In the monocular case, we construct the graph $\mathbf{G}=(\mathbf{V}, \mathbf{E})$ by taking each pixel as a node and define the edge weight $w_{i j}$ between node $i$ and $j$ as the product of a feature similarity term and spatial proximity term:

$$
w_{i j}=e^{\frac{-\|\boldsymbol{F}(i)-\boldsymbol{F}(j)\|_{2}^{2}}{\sigma_{I}}} * \begin{cases}e^{\frac{-\|\boldsymbol{X}(i)-\boldsymbol{X}(j)\|_{2}^{2}}{\sigma_{X}}} & \text { if }\|\boldsymbol{X}(i)-\boldsymbol{X}(j)\|_{2}<r \\ 0 & \text { otherwise, }\end{cases}
$$

where $X(i)$ is the spatial location of node $i$, and $F(i)$ is a feature vector based on intensity, color, or texture information at that node defined as:

- $F(i)=1$, in the case of segmenting point sets,
- $F(i)=I(i)$, the intensity value, for segmenting brightness images,

- $\quad \boldsymbol{F}(i)=[v, v \cdot s \cdot \sin (h), v \cdot s \cdot \cos (h)](i)$, where $h, s, v$ are the HSV values, for color segmentation,

- $\boldsymbol{F}(i)=\left[\left|\boldsymbol{I} * f_{1}\right|, \ldots,\left|\boldsymbol{I} * f_{n}\right|\right](i)$, where the $f_{i}$ are DOOG filters at various scales and orientations as used in [16], in the case of texture segmentation.

Note that the weight $w_{i j}=0$ for any pair of nodes $i$ and $j$ that are more than $r$ pixels apart.

We first tested our grouping algorithm on spatial point sets. Fig. 5 shows a point set and the segmentation result. The normalized cut criterion is indeed able to partition the point set in a desirable way.

Figs. 4, 6, 7, and 8 show the result of our segmentation algorithm on various brightness images. Figs. 6 and 7 are synthetic images with added noise. Figs. 4 and 8 are natural images. Note that the "objects" in Fig. 8 have rather illdefined boundaries, which would make edge detection perform poorly. Fig. 9 shows the segmentation on a color image, reproduced in gray scale in these transactions. The original image and many other examples can be found at web site http://www.cs.berkeley.edu/ jshi/Grouping.

Note that, in all these examples, the algorithm is able to extract the major components of scene while ignoring small intracomponent variations. As desired, recursive partitioning can be used to further decompose each piece.

Fig. 10 shows preliminary results on texture segmentation for a natural image of a zebra against a background. Note that the measure we have used is orientation-variant and, therefore, parts of the zebra skin with different stripe orientation should be marked as separate regions.

In the motion case, we will treat the image sequence as a spatiotemporal data set. Given an image sequence, a weighted graph is constructed by taking each pixel in the image sequence as a node and connecting pixels that are in the spatiotemporal neighborhood of each other. The weight on each graph edge is defined as: 


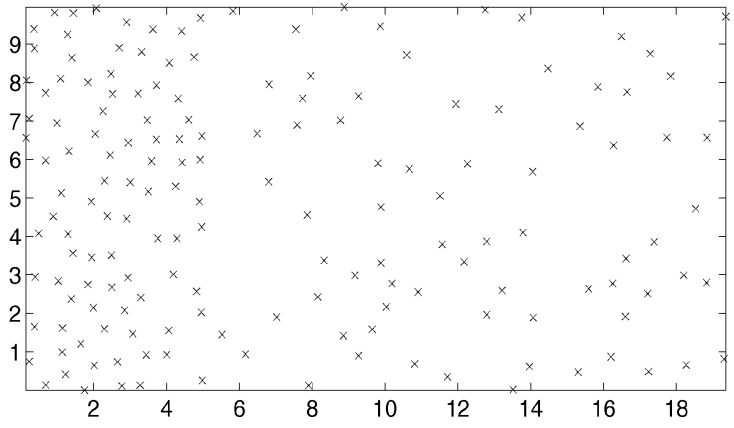

(a)

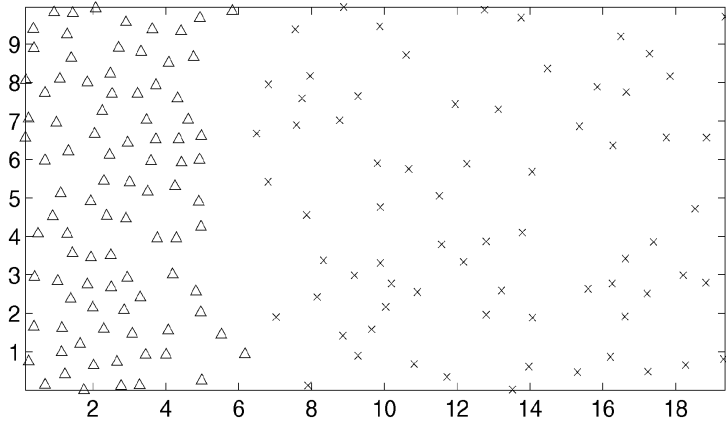

(b)

Fig. 5. (a) Point set generated by two Poisson processes, with densities of 2.5 and 1.0 on the left and right clusters respectively, (b) $\triangle$ and $\times$ indicate the partition of point set in (a). Parameter settings: $\sigma_{X}=5, r=3$.

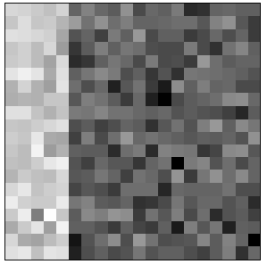

(a)

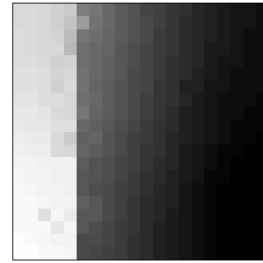

(b)

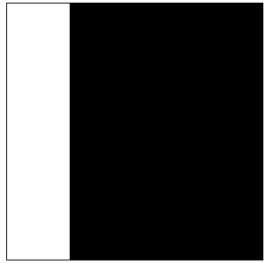

(c)

Fig. 6. (a) A synthetic image showing a noisy "step" image. Intensity varies from 0 to 1 , and Gaussian noise with $\sigma=0.2$ is added. Subplot (b) shows the eigenvector with the second smallest eigenvalue and subplot (c) shows the resulting partition.

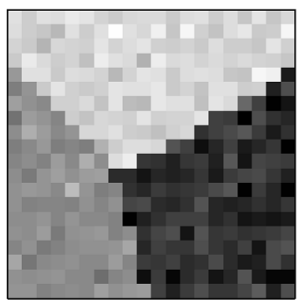

(a)

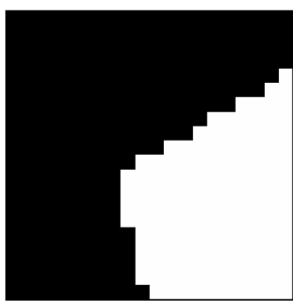

(b)

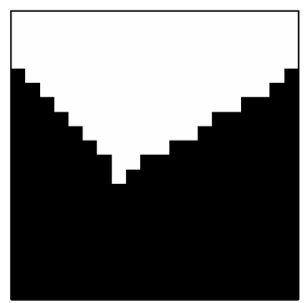

(c)

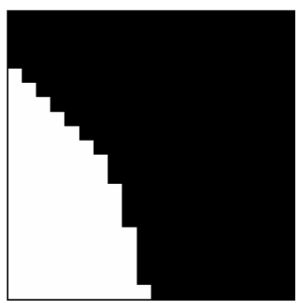

(d)

Fig. 7. (a) A synthetic image showing three image patches forming a junction. Image intensity varies from 0 to 1 and Gaussian noise with $\sigma=0.1$ is added. (b)-(d) show the top three components of the partition.

$$
w_{i j}= \begin{cases}e^{\frac{-\mathbf{d}_{m}(i, j)^{2}}{\sigma_{m}^{2}}} & \text { if }\|X(i)-X(j)\|_{2}<r \\ 0 & \text { otherwise, }\end{cases}
$$

where $\mathbf{d}(i, j)$ is the "motion distance" between two pixels $i$ and $j$. Note that $X_{i}$ in this case represents the spatialtemporal position of pixel $i$.

To compute this "motion distance," we will use a motion feature called motion profile. By motion profile we seek to estimate the probability distribution of image velocity at each pixel. Let $I^{t}(\mathbf{X})$ denote a image window centered at the pixel at location $\mathbf{X} \in R^{2}$ at time $t$. We denote by $P_{i}(\mathbf{d x})$ the motion profile of an image patch at node $i, \boldsymbol{I}^{t}\left(\mathbf{X}_{i}\right)$, at time $t$ corresponding to another image patch $I^{t+1}\left(\mathbf{X}_{i}+\mathbf{d x}\right)$ at time $t+1$. $P_{i}(\mathbf{d x})$ can be estimated by first computing the similarity $S_{i}(\mathbf{d x})$ between $\boldsymbol{I}^{t}\left(\mathbf{X}_{i}\right)$ and $\boldsymbol{I}^{t+1}\left(\mathbf{X}_{i}+\mathbf{d x}\right)$ and normalizing it to get a probability distribution:

$$
P_{i}(\mathbf{d x})=\frac{S_{i}(\mathbf{d x})}{\sum_{\mathbf{d x}} S_{i}(\mathbf{d x})} .
$$

There are many ways one can compute similarity between two image patches; we will use a measure that is based on the sum of squared differences (SSD):

$$
\begin{aligned}
S_{i}(\mathbf{d x})=\exp ( & -\sum_{w}\left(\boldsymbol{I}^{t}\left(\mathbf{X}_{i}+\mathbf{w}\right)\right. \\
& \left.\left.-\boldsymbol{I}^{t+1}\left(\mathbf{X}_{i}+\mathbf{d} \mathbf{x}+\mathbf{w}\right)\right)^{2} / \sigma_{s s d}^{2}\right)
\end{aligned}
$$

where $\mathbf{w} \in R^{2}$ is within a local neighborhood of image patch $\boldsymbol{I}^{t}\left(\mathbf{X}_{i}\right)$. The "motion distance" between two image pixels is then defined as one minus the cross-correlation of the motion profiles:

$$
\mathbf{d}(i, j)=1-\sum_{\mathbf{d x}} P_{i}(\mathbf{d x}) P_{j}(\mathbf{d} \mathbf{x}) .
$$

In Fig. 11, we show results of the normalized cut algorithm on a synthetic random dot motion sequence and a indoor motion sequence, respectively. For more elaborate discussion on motion segmentation using normalized cut, as well as how to segment and track over long image sequences, readers might want to refer to our paper [21].

\subsection{Computation Time}

As we saw from Section 3.1, the running time of the normalized cut algorithm is $O(m n)$, where $n$ is the number 


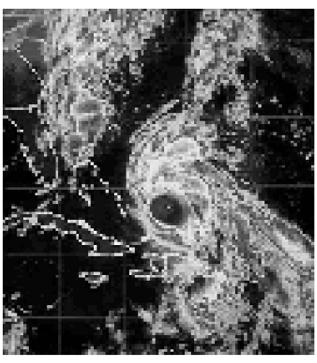

(a)

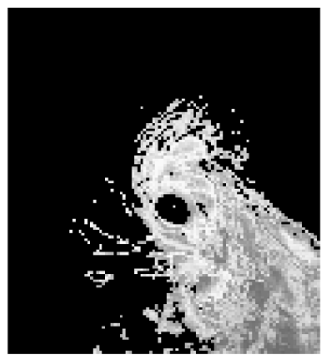

(b)

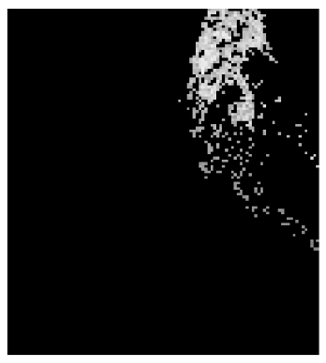

(c)

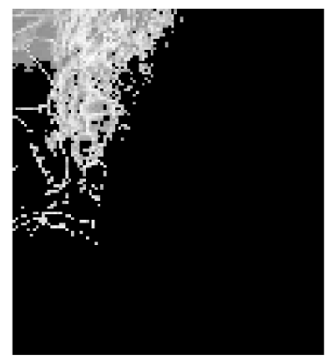

(d)

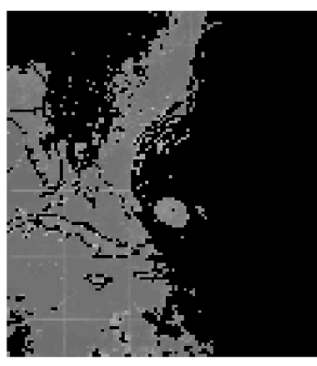

$(e)$

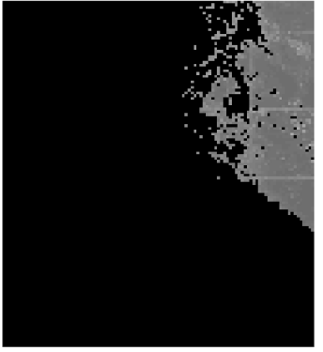

(f)

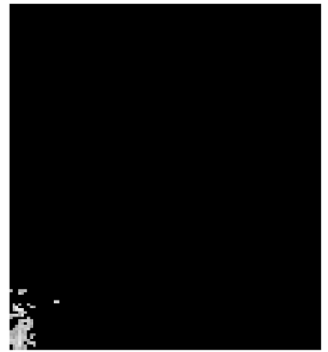

(g)

Fig. 8. (a) shows a $126 \times 106$ weather radar image. (b)-(g) show the components of the partition with $N$ cut value less than 0.08 . Parameter setting: $\sigma_{I}=0.007, \sigma_{x}=15.0, r=10$.

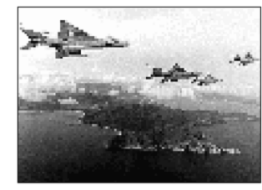

(a)

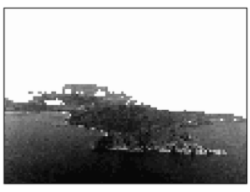

(b)

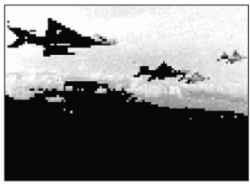

(c)

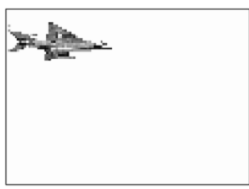

(d)

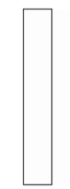

$\Leftrightarrow$

(e)

Fig. 9. (a) shows a $77 \times 107$ color image. (b)-(e) show the components of the partition with $N$ cut value less than 0.04 . Parameter settings: $\sigma_{I}=0.01$, $\sigma_{X}=4.0, r=5$.

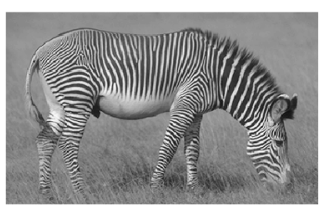

(a)

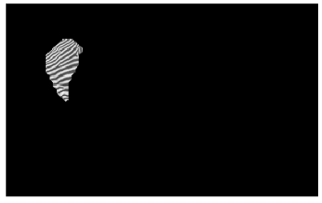

(e)

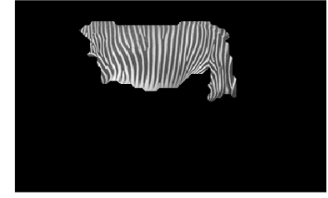

(b)

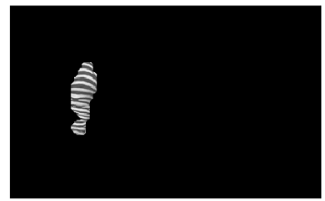

(f)

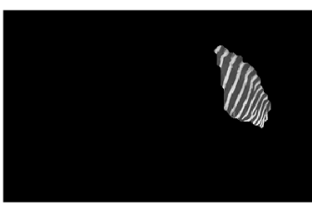

(c)

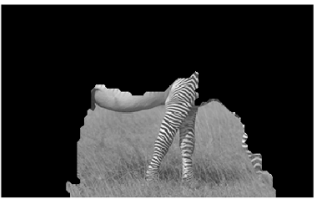

(g)

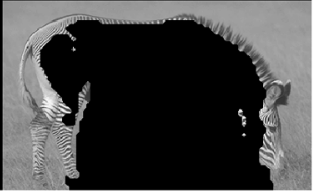

(d)

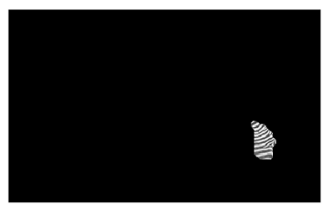

(h)

Fig. 10. (a) shows an image of a zebra. The remaining images show the major components of the partition. The texture features used correspond to convolutions with DOOG filters [16] at six orientations and five scales.

of pixels and $m$ is the number of steps Lanczos takes to converge. On the $100 \times 120$ test images shown here, the normalized cut algorithm takes about 2 minutes on Intel Pentium 200MHz machines.

A multiresolution implementation can be used to reduce this running time further on larger images. In our current experiments, with this implementation, the running time on a $300 \times 400$ image can be reduced to about 20 seconds on Intel Pentium $300 \mathrm{MHz}$ machines. Furthermore, the bottleneck of the computation, a sparse matrix-vector multiplication step, can be easily parallelized taking advantage of future computer chip designs.

In our current implementation, the sparse eigenvalue decomposition is computed using the LASO2 numerical package developed by Scott.

\subsection{Choice of Graph Edge Weight}

In the examples shown here, we used an exponential function of the form of $w(x)=e^{-(d(x) / \sigma)^{2}}$ on the weighted graph edge with feature similarity of $d(x)$. The value of $\sigma$ is 


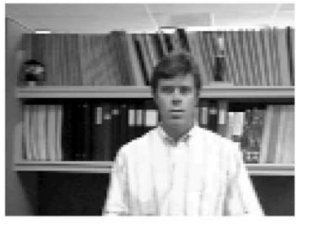

(a)

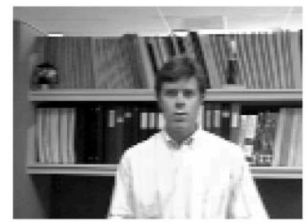

(b)

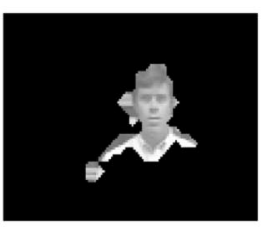

(c)

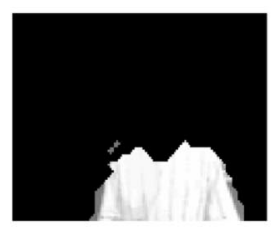

(d)

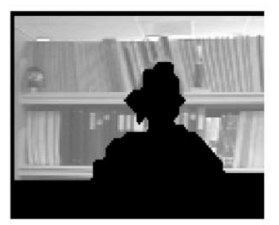

(e)

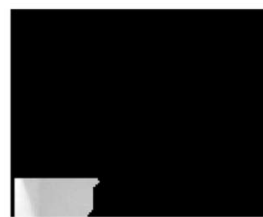

(f)

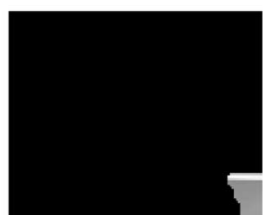

(g)

Fig. 11. Subimages (a) and (b) show two frames of an image sequence. Segmentation results on this two frame image sequence are shown in subimages (c) to (g). Segments in (c) and (d) correspond to the person in the foreground and segments in (e) to (g) correspond to the background. The reason that the head of the person is segmented away from the body is that, although they have similar motion, their motion profiles are different. The head region contains $2 \mathrm{D}$ textures and the motion profiles are more peaked, while, in the body region, the motion profiles are more spread out. Segment $(e)$ is broken away from $(f)$ and $(g)$ for the same reason.

typically set to 10 to 20 percent of the total range of the feature distance function $d(x)$. The exponential weighting function is chosen here for its relative simplicity, as well as neutrality, since the focus of this paper is on developing a general segmentation procedure, given a feature similarity measure. We found this choice of weight function is quite adequate for typical image and feature spaces. Section 6.1 shows the effect of using different weighting functions and parameters on the output of the normalized cut algorithm.

However, the general problem of defining feature similarity incorporating a variety of cues is not a trivial one. The grouping cues could be of different abstraction levels and types and they could be in conflict with each other. Furthermore, the weighting function could vary from image region to image region, particularly in a textured image. Some of these issues are addressed in [15].

\section{Relationship to Spectral Graph Theory}

The computational approach that we have developed for image segmentation is based on concepts from spectral graph theory. The core idea is to use matrix theory and linear algebra to study properties of the incidence matrix, $\mathbf{W}$, and the Laplacian matrix, $\mathbf{D}-\mathbf{W}$, of the graph and relate them back to various properties of the original graph. This is a rich area of mathematics and the idea of using eigenvectors of the Laplacian for finding partitions of graphs can be traced back to Cheeger [4], Donath and Hoffman [7], and Fiedler [9]. This area has also seen contributions by theoretical computer scientists [1], [3], [22], [23]. It can be shown that our notion of normalized cut is related by a constant factor to the concept of conductance in [22].

For a tutorial introduction to spectral graph theory, we recommend the recent monograph by Chung [5]. In this monograph, Chung proposes a "normalized" definition of the Laplacian, as $\mathbf{D}^{-\frac{1}{2}}(\mathbf{D}-\mathbf{W}) \mathbf{D}^{-\frac{1}{2}}$. The eigenvectors for this "normalized" Laplacian, when multiplied by $\mathbf{D}^{-\frac{1}{2}}$, are exactly the generalized eigenvectors we used to compute normalized cut. Chung points out that the eigenvalues of this "normalized" Laplacian relate well to graph invariants for general graph in ways that eigenvalues of the standard Laplacian have failed to do.

Spectral graph theory provides us some guidance on the goodness of the approximation to the normalized cut provided by the second eigenvalue of the normalized Laplacian. One way is through bounds on the normalized Cheeger constant [5] which, in our terminology, can be defined as

$$
h_{G}=\inf \frac{\operatorname{cut}(A, B)}{\min (\operatorname{assoc}(A, V), \operatorname{assoc}(B, V))} .
$$

The eigenvalues of (6) are related to the Cheeger constant by the inequality [5]:

$$
2 h_{G} \geq \lambda_{1}>\frac{h_{G}^{2}}{2} .
$$

Earlier work on spectral partitioning used the second eigenvectors of the Laplacian of the graph defined as $\mathbf{D}-\mathbf{W}$ to partition a graph. The second smallest eigenvalue of $\mathbf{D}-\mathbf{W}$ is sometimes known as the Fiedler value. Several results have been derived relating the ratio cut and the Fiedler value. A ratio cut of a partition of $V, P=(A, V-A)$, which, in fact, is the standard definition of the Cheeger constant, is defined as $\frac{\operatorname{cut}(A, V-A)}{\min (|A|,|V-A|)}$. It was shown that if the Fiedler value is small, partitioning the graph based on the Fiedler vector will lead to good ratio cut [1], [23]. Our derivation in Section 2.1 can be adapted (by replacing the matrix $\mathbf{D}$ in the denominators by the identity matrix $\mathbf{I}$ ) to show that the Fiedler vector is a real valued solution to the problem of $\min _{A \subset V} \frac{\operatorname{cut}(A, V-A)}{|A|}+\frac{\operatorname{cut}(V-A, A)}{|V-A|}$, which we can call the average cut.

Although average cut looks similar to the normalized cut, average cut does not have the important property of having a 
simple relationship to the average association, which can be analogously defined as $\frac{\operatorname{assoc}(A, A)}{|A|}+\frac{\operatorname{assoc}(V-A, V-A)}{|V-A|}$. Consequently, one cannot simultaneously minimize the disassociation across the partitions while maximizing the association within the groups. When we applied both techniques to the image segmentation problem, we found that the normalized cut produces better results in practice. There are also other explanations why the normalized cut has better behavior from graph theoretical point of view, as pointed out by Chung [5].

Our work, originally presented in [20], represents the first application of spectral partitioning to computer vision or image analysis. There is, however, one application area that has seen substantial application of spectral partitioning-the area of parallel scientific computing. The problem there is to balance the workload over multiple processors taking into account communication needs. One of the early papers is [18]. The generalized eigenvalue approach was first applied to graph partitioning by [8] for dynamically balancing computational load in a parallel computer. Their algorithm is motivated by [13]'s paper on representing a hypergraph in a Euclidean space.

The normalized cut criteria is also closely related to key properties of a Markov Random Walk. The similarity matrix W can be normalized to define a probability transition matrix $\mathbf{P}$ of a random walk on the pixels. It can be shown that the conductance [22] of this random walk is the normalized cut value and the normalized cut vectors of (12) are exactly the right eigenvectors of $\mathbf{P}$.

\subsection{A Physical Interpretation}

As one might expect, a physical analogy can be set up for the generalized eigenvalue system (6) that we used to approximate the solution of normalized cut. We can construct a spring-mass system from the weighted graph by taking graph nodes as physical nodes and graph edges as springs connecting each pair of nodes. Furthermore, we will define the graph edge weight as the spring stiffness and the total edge weights connecting to a node as its mass.

Imagine what would happen if we were to give a hard shake to this spring-mass system, forcing the nodes to oscillate in the direction perpendicular to the image plane. Nodes that have stronger spring connections among them will likely oscillate together. As the shaking becomes more violent, weaker springs connecting to this group of node will be overstretched. Eventually, the group will "pop" off from the image plane. The overall steady state behavior of the nodes can be described by its fundamental mode of oscillation. In fact, it can be shown that the fundamental modes of oscillation of this spring mass system are exactly the generalized eigenvectors of (6).

Let $k_{i j}$ be the spring stiffness connecting nodes $i$ and $j$. Define $\mathbf{K}$ to be the $n \times n$ stiffness matrix, with $\mathbf{K}(i, i)=$ $\sum_{i} k_{i j}$ and $\mathbf{K}(i, j)=-k_{i j}$. Define the diagonal $n \times n$ mass matrix $\mathbf{M}$ as $\mathbf{M}(i, i)=\sum_{i} k_{i j}$. Let $\mathbf{x}(t)$ be the $n \times 1$ vector describing the motion of each node. This spring-mass dynamic system can be described by:

$$
\mathbf{K} \mathbf{x}(t)=-\mathbf{M}(\ddot{\mathbf{x}})(t) .
$$

Assuming the solution takes the form of $\mathbf{x}(t)=v_{k} \cos \left(\omega_{k} t+\theta\right)$, the steady state solutions of this spring-mass system satisfy:

$$
\mathbf{K} \mathbf{v}_{k}=\omega_{k}^{2} \mathbf{M} \mathbf{v}_{k},
$$

analogous to (6) for normalized cut.

Each solution pair $\left(\omega_{k}, \mathbf{v}_{k}\right)$ of (21) describes a fundamental mode of the spring-mass system. The eigenvectors $\mathbf{v}_{k}$ give the steady state displacement of the oscillation in each mode and the eigenvalues $\omega_{k}^{2}$ give the energy required to sustain each mode of oscillation. Therefore, finding graph partitions that have small normalized cut values is, in effect, the same as finding a way to "pop" off image regions with minimal effort.

\section{Relationship to Other Graph Theoretic ApProaches to IMAge SEgmentation}

In the computer vision community, there has been some been previous work on image segmentation formulated as a graph partition problem. Wu and Leahy [25] use the minimum cut criterion for their segmentation. As mentioned earlier, our criticism of this criterion is that it tends to favor cutting off small regions, which is undesirable in the context of image segmentation. In an attempt to get more balanced partitions, Cox et al. [6] seek to minimize the ratio $\frac{\operatorname{cut}(A, V-A)}{\text { weight }(A)}, A \subset V$, where weight $(A)$ is some function of the set $A$. When weight $(A)$ is taken to be the sum of the elements in $A$, we see that this criterion becomes one of the terms in the definition of average cut above. Cox et al. use an efficient discrete algorithm to solve their optimization problem assuming the graph is planar.

Sarkar and Boyer [19] use the eigenvector with the largest eigenvalue of the system $\mathbf{W} x=\lambda x$ for finding the most coherent region in an edge map. Using a similar derivation as in Section 2.1, we can see that the first largest eigenvector of their system approximates $\min _{A \subset V} \frac{\operatorname{assoc}(A, A)}{|A|}$ and the second largest eigenvector approximates $\min _{A \subset V, B \subset V} \frac{\operatorname{assoc}(A, A)}{|A|}+\frac{\operatorname{assoc}(B, B)}{|B|}$. However, the approximation is not tight and there is no guarantee that $A+B=V$. As we will see later in the section, this situation can happen quite often in practice. Since this algorithm is essentially looking for clusters that have tight within-grouping similarity, we will call this criteria average association.

\subsection{Comparison with Related Eigenvector-Based Methods}

The normalized cut formulation has a certain resemblance to the average cut, the standard spectral graph partitioning, as well as average association formulation. All three of these algorithms can be reduced to solving certain eigenvalue systems. How are they related to each other?

Fig. 12 summarizes the relationship between these three algorithms. On one hand, both the normalized cut and the average cut algorithm are trying to find a "balanced partition" of a weighted graph, while, on the other hand, 


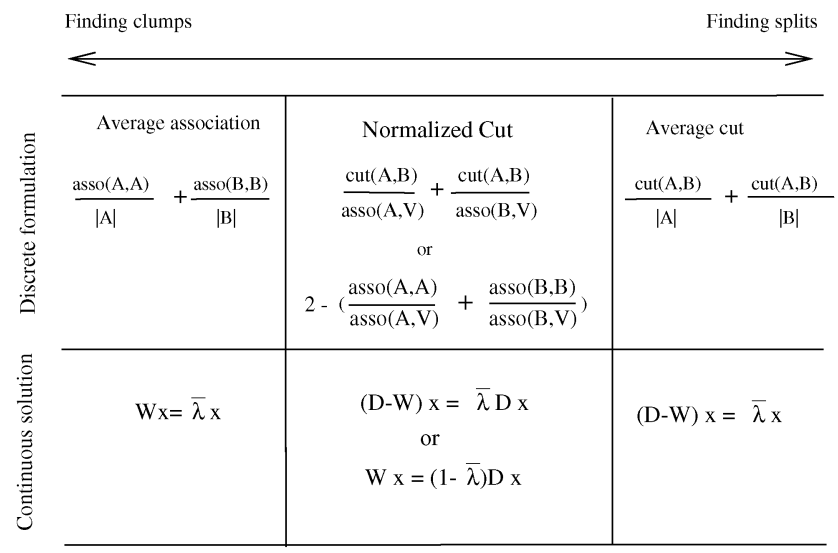

Fig. 12. Relationship between normalized cut and other eigenvector-based partitioning techniques. Compared to the average cut and average association formulation, normalized cut seeks a balance between the goal of finding clumps and finding splits.

the normalized association and the average association are trying to find "tight" clusters in the graph. Since the normalized association is exactly $2-$ ncut, the normalized cut value, the normalized cut formulation seeks a balance between the goal of clustering and segmentation. It is, therefore, not too surprising to see that the normalized cut vector can be approximated with the generalized eigenvector of $(\mathbf{D}-\mathbf{W}) \mathbf{x}=\lambda \mathbf{D} \mathbf{x}$, as well as that of $\mathbf{W} \mathbf{x}=\lambda \mathbf{D} \mathbf{x}$.

Judging from the discrete formulations of these three grouping criteria, it can be seen that the average association, $\frac{\operatorname{assoc}(A, A)}{|A|}+\frac{\operatorname{assoc}(B, B)}{|B|}$, has a bias for finding tight clusters. Therefore, it runs the risk of becoming too greedy in finding small, but tight, clusters in the data. This might be perfect for data that are Gaussian distributed. However, for typical data in the real world that are more likely to be made up of a mixture of various different types of distributions, this bias in grouping will have undesired consequences, as we shall illustrate in the examples below.

For average cut, $\frac{\operatorname{cut}(A, B)}{|A|}+\frac{\operatorname{cut}(A, B)}{|B|}$, the opposite problem arises-one cannot ensure the two partitions computed will have tight within-group similarity. This becomes particularly problematic if the dissimilarity among the different groups varies from one to another, or if there are several possible partitions all with similar average cut values.

To illustrate these points, let us first consider a set of randomly distributed data in 1D shown in Fig. 13. The 1D data is made up by two subsets of points, one randomly distributed from 0 to 0.5 and the other from 0.65 to 1.0. Each data point is taken as a node in the graph and the weighted graph edge connecting two points is defined to be inversely proportional to the distance between two nodes. We will use three monotonically decreasing weighting functions, $w(x)=f(d(x))$, defined on the distance function, $d(x)$, with different rate of fall-off. The three weighting functions are plotted in Figs. 14a, 15a, and 16a.

The first function, $w(x)=e^{-\left(\frac{d(x)}{0.1}\right)^{2}}$, plotted in Fig. 14a, has the fastest decreasing rate among the three. With this weighting function, only close-by points are connected, as shown in the graph weight matrix $\mathbf{W}$ plotted in Fig. 14b. In this case, average association fails to find the right partition.
Instead, it focuses on finding small clusters in each of the two main subgroups.

The second function, $w(x)=1-d(x)$, plotted in Fig. 15a, has the slowest decreasing rate among the three. With this weighting function, most points have some nontrivial connections to the rest. To find a cut of the graph, a number of edges with heavy weights have to be removed. In addition, the cluster on the right has less within-group similarity comparing with the cluster on the left. In this case, average cut has trouble deciding on where to cut.

The third function, $w(x)=e^{-\frac{d(x)}{0.2}}$, plotted in Fig. 16a, has a moderate decreasing rate. With this weighting function, the nearby point connections are balanced against far-away point connections. In this case, all three algorithms perform well with normalized cut, producing a clearer solution than the two other methods.

These problems, illustrated in Figs. 14, 15, and 16, in fact are quite typical in segmenting real natural images. This is particularly true in the case of texture segmentation. Different texture regions often have very different withingroup similarity or coherence. It is very difficult to predetermine the right weighting function on each image region. Therefore, it is important to design a grouping algorithm that is more tolerant to a wide range of weighting functions. The advantage of using normalized cut becomes more evident in this case. Fig. 17 illustrates this point on a natural texture image shown previously in Fig. 10.

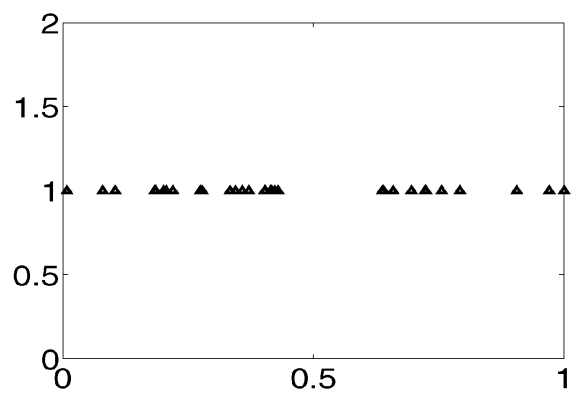

Fig. 13. A set of randomly distributed points in 1D. The first 20 points are randomly distributed from 0.0 to 0.5 and the remaining 12 points are randomly distributed from 0.65 to 1.0. Segmentation result of these points with different weighting functions are shown in Figs. 14, 15, and 16. 
Normalized Cut:

$(D-W) x=\lambda D x$

$W x=(1-\lambda) D x$

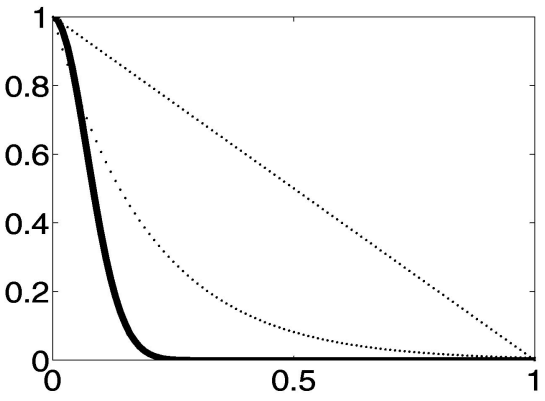

(a)

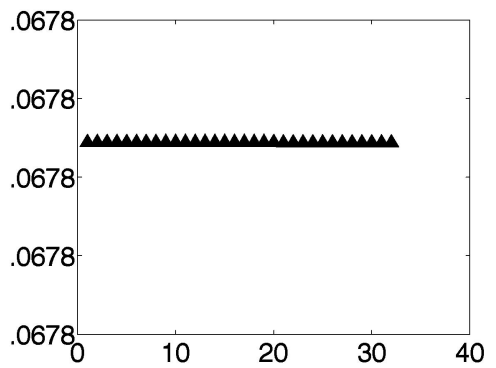

Average Cut:

$(D-W) x=\lambda x$

Average Association:

$$
W x=\lambda x
$$

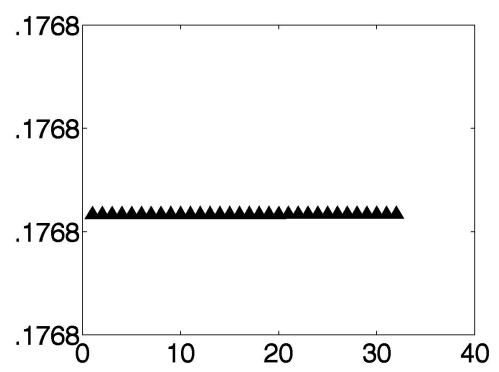

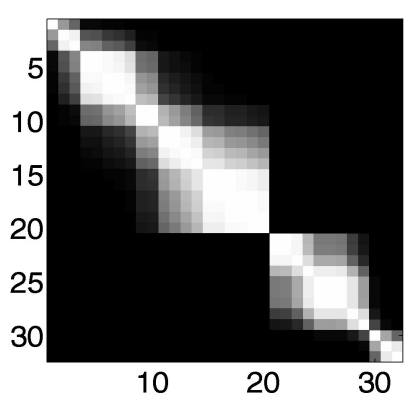

(b)
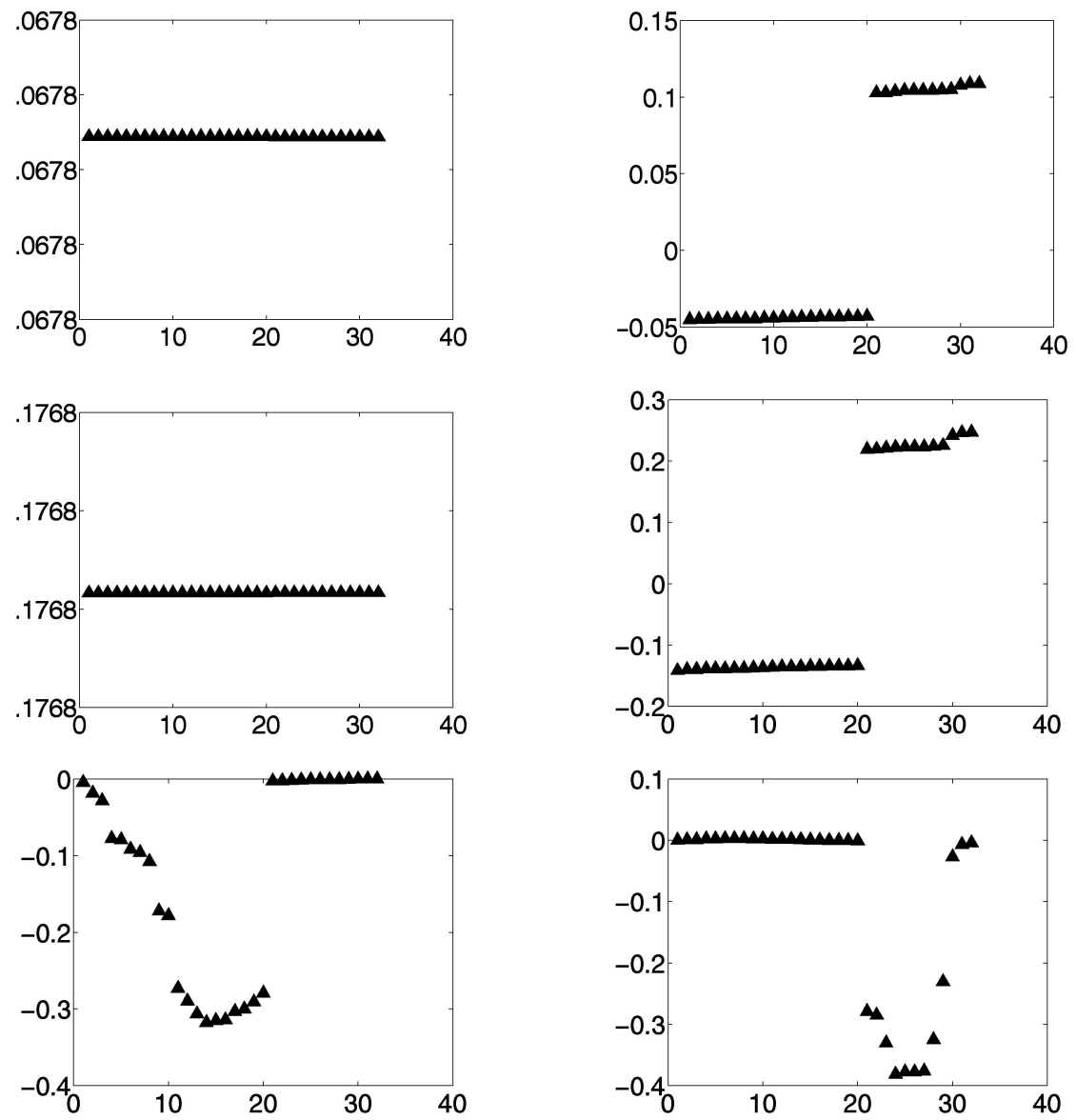

(c)

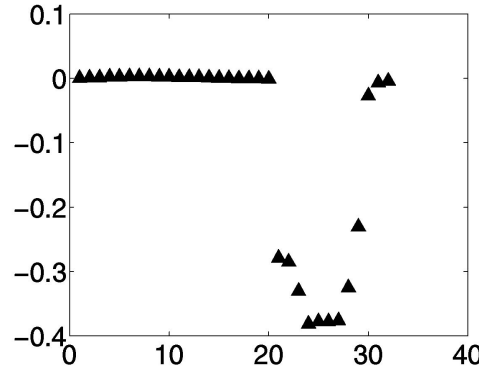

(d)

Fig. 14. A weighting function with fast rate of fall-off: $w(x)=e^{-\left(\frac{d(x)}{0.1}\right)^{2}}$, shown in subplot (a) in solid line. The dotted lines show the two alternative weighting functions used in Figs. 15 and 16. Subplot (b) shows the corresponding graph weight matrix $W$. The two columns (c) and (d) below show the first, and second extreme eigenvectors for the Normalized cut (row 1), Average cut (row 2), and Average association (row 3). For both normalized cut and average cut, the smallest eigenvector is a constant vector as predicted. In this case, both normalized cut and average cut perform well, while the average association fails to do the right thing. Instead, it tries to pick out isolated small clusters.

\section{CONCLUSION}

In this paper, we developed a grouping algorithm based on the view that perceptual grouping should be a process that aims to extract global impressions of a scene and provides a hierarchical description of it. By treating the grouping problem as a graph partitioning problem, we proposed the normalized cut criteria for segmenting the graph. Normalized cut is an unbiased measure of disassociation between subgroups of a graph and it has the nice property that minimizing normalized cut leads directly to maximizing the normalized association, which is an unbiased measure for total association within the subgroups. In finding an efficient algorithm for computing the minimum normalized cut, we showed that a generalized eigenvalue system provides a real valued solution to our problem.

A computational method based on this idea has been developed and applied to segmentation of brightness, color, and texture images. Results of experiments on real and synthetic images are very encouraging and illustrate that the normalized cut criterion does indeed satisfy our initial goal of extracting the "big picture" of a scene. 


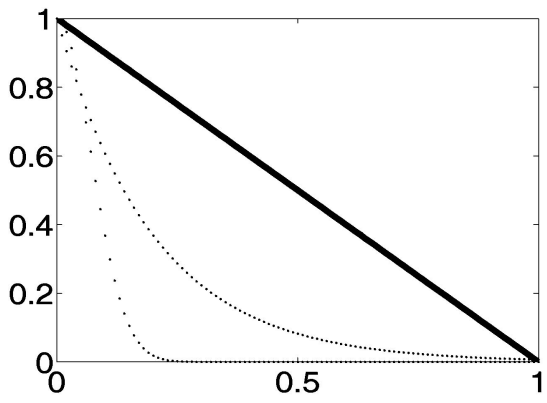

(a)

Normalized Cut:

$(D-W) x=\lambda D x$

$W x=(1-\lambda) D x$

Average Cut:

$(D-W) x=\lambda x$

Average Association:

$$
W x=\lambda x
$$
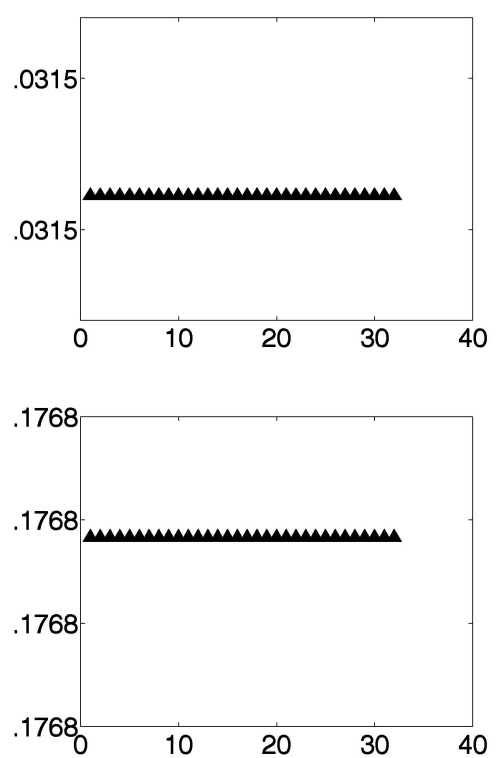

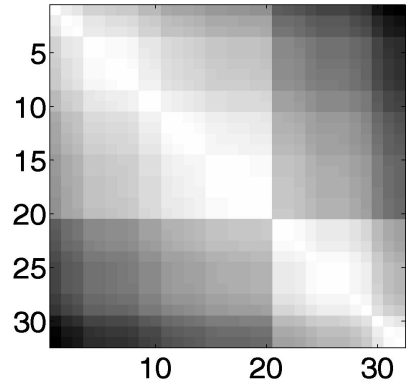

(b)
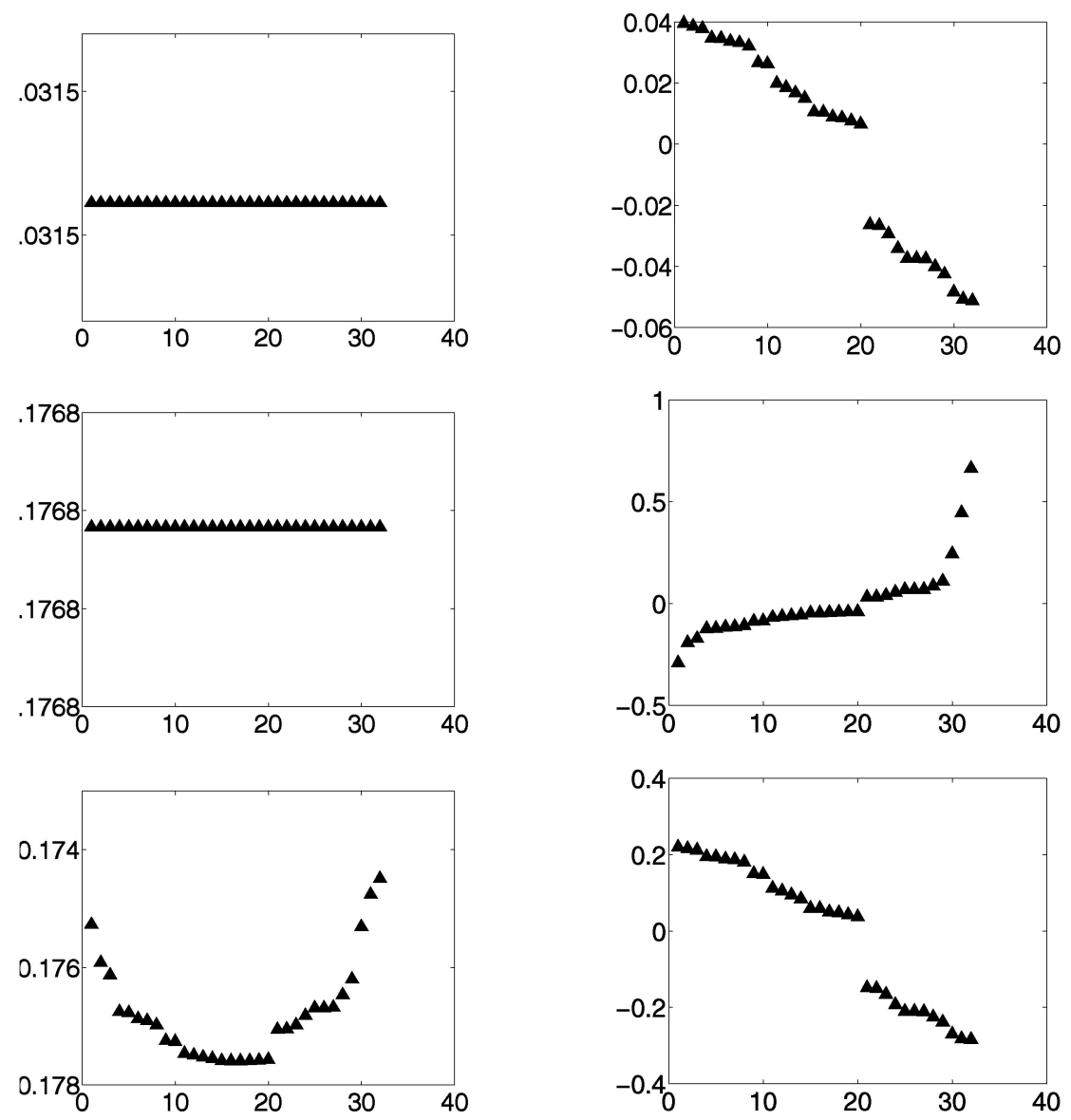

(c)

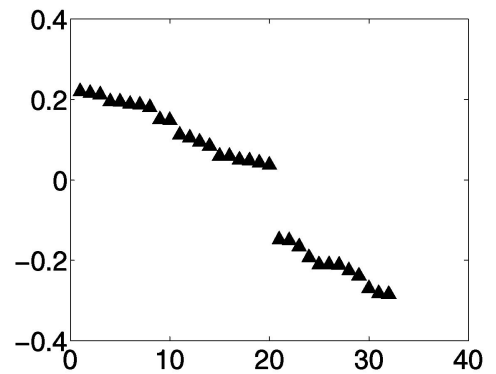

(d)

Fig. 15. A weighting function with slow rate of fall-off: $w(x)=1-d(x)$, shown in subplot (a) in solid line. The dotted lines show the two alternative weighting functions used in Figs. 14 and 16. Subplot (b) shows the corresponding graph weight matrix $W$. The two columns (c) and (d) below show the first, and second extreme eigenvectors for the Normalized cut (row 1), Average cut (row 2), and Average association (row 3 ). In this case, both normalized cut and average association give the right partition, while the average cut has trouble deciding on where to cut.

\section{APPENDIX}

\section{NP-Completeness Proof for Normalized Cut}

Proposition 1 [Papadimitrou 97]. Normalized Cut (NCUT)

for a graph on regular grids is NP-complete.

Proof. We shall reduce NCUT on regular grids from

\section{PARTITION:}

- Given integers $x_{1}, x_{2}, \ldots, x_{n}$ adding to $2 k$, is there a subset adding to $k$ ?
We construct a weighted graph on a regular grid that has the property that it will have a small enough normalized cut if and only if we can find a subset from $x_{1}, x_{2}, \ldots, x_{n}$ adding to $k$. Fig. 18a shows the graph and Fig. $18 \mathrm{~b}$ shows the form that a partition that minimizes the normalized cut must take.

In comparison to the integers $x_{1}, x_{2}, \ldots, x_{n}, M$ is much larger, $M>2 k^{2}$, and $a$ is much smaller, $0<a<1 / n$. We ask the question 


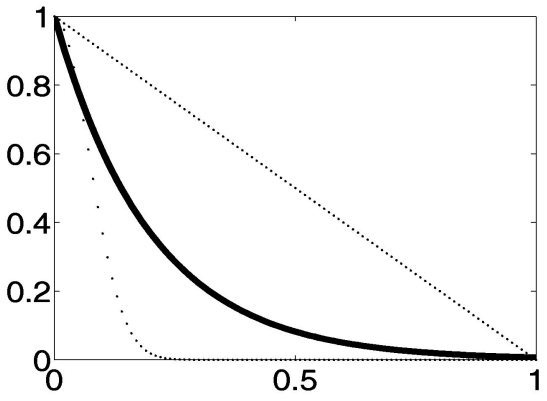

(a)

Normalized Cut:

$(D-W) x=\lambda D x$

$W x=(1-\lambda) D x$
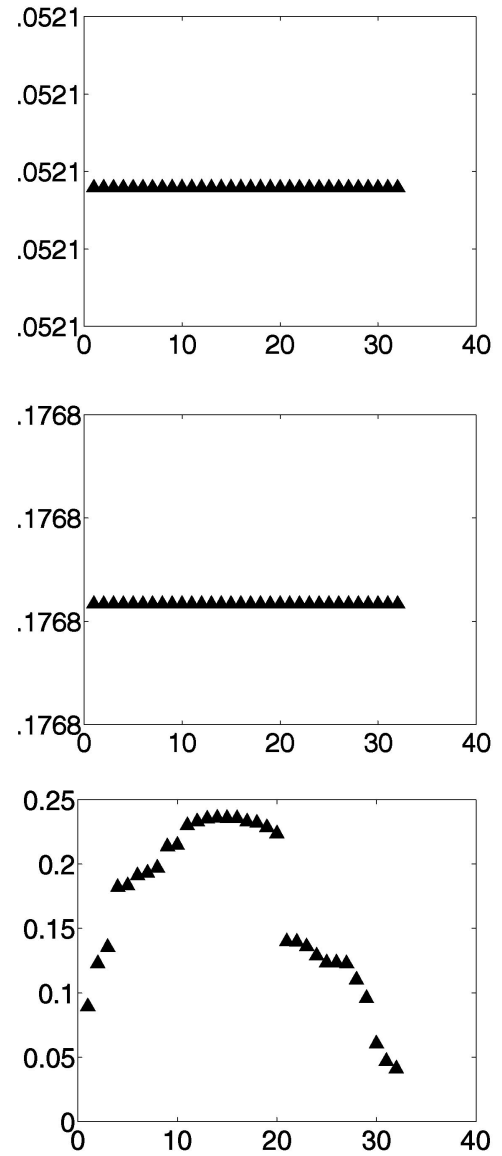

(c)

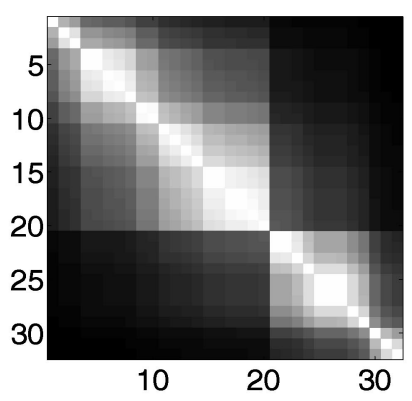

(b)
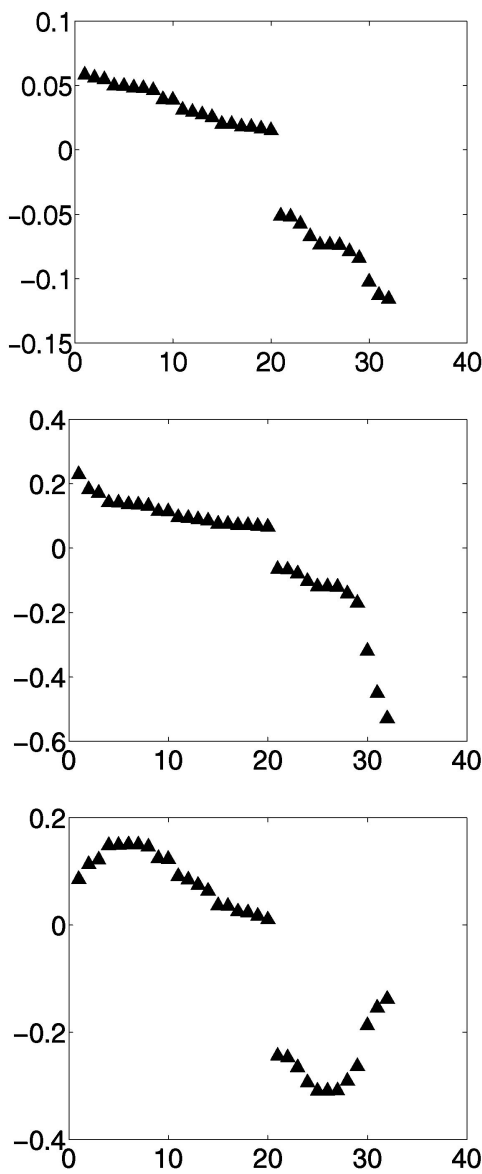

(d)

Fig. 16. A weighting function with medium rate of fall-off: $w(x)=e^{-\frac{d(x)}{0.2}}$, shown in subplot (a) in solid line. The dotted lines show the two alternative weighting functions used in Figs. 14 and 15. Subplot (b) shows the corresponding graph weight matrix $W$. The two columns (c) and (d) below show the first and second extreme eigenvectors for the Normalized cut (row 1), Average cut (row 2), and average association (row 3 ). All three of these algorithms perform satisfactorily in this case, with normalized cut producing a clearer solution than the other two cuts.

- Is there a partition with $N c u t$ value less than $\frac{4 a n}{c-1 / c}$, where $c$ is half the sum of edge weights in the graph, $c=2 M(n+1)+k+3 a n$.

We shall see that a good Ncut partition of the graph must separate the left and right columns. In particular, if and only if there is a subset $S_{1}=\left\{x_{1}, \ldots, x_{m}\right\}$ adding to $k$, by taking the corresponding edges in the middle column to be in one side of the partition, as illustrated in Fig. 18b, we achieve an $N c u t$ value less than $\frac{4 a n}{c-1 / c}$. For all other partitions, the Ncut value will be bounded below by $\frac{4 a n}{c-1 / c}$.

First, let us show that the cut illustrated in Fig. 18b, where each side has a subset of middle column edges $x_{1}, x_{2}, \ldots, x_{n}$ that add up to $k$, does have $N c u t$ value less than $\frac{4 a n}{c-1 / c}$. Let the $n c u t^{*}$ be the $N c u t$ value for this cut. By using the formula for Ncut (2.2), we can see that 


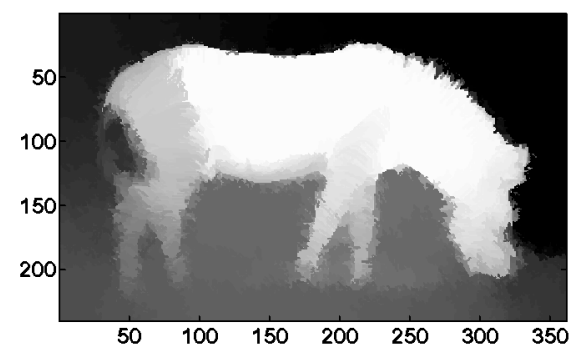

(a)

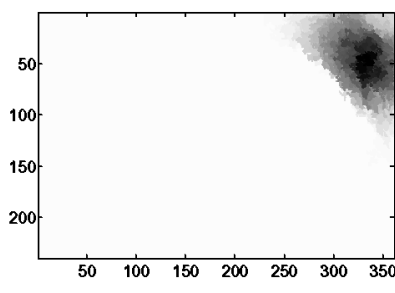

(b)

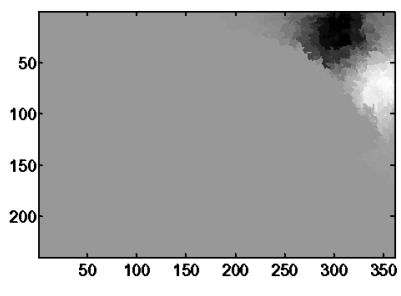

(c)

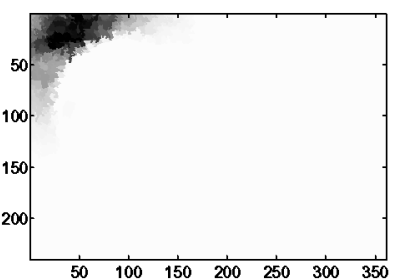

(d)

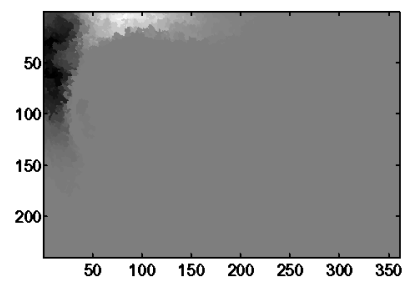

(e)

Fig. 17. Normalized cut and average association result on the zebra image in Fig. 10. Subplot (a) shows the second largest eigenvector of $W \mathbf{x}=\lambda D \mathbf{x}$, approximating the normalized cut vector. Subplots (b)-(e) show the first to fourth largest eigenvectors of $W \mathbf{x}=\lambda \mathbf{x}$, approximating the average association vector, using the same graph weight matrix. In this image, pixels on the zebra body have, on average, lower degree of coherence than the pixels in the background. The average association, with its tendency to find tight clusters, partitions out only small clusters in the background. The normalized cut algorithm, having to balance the goal of clustering and segmentation, finds the better partition in this case.

$$
n c u t^{*}=\frac{4 a n}{2 c+2 a n\left(2 k_{1}-1\right)}+\frac{4 a n}{2 c-2 a n\left(2 k_{1}-1\right)},
$$

where $c$ is half the total edge weights in the graph, $c=2 M(n+1)+k+3 a n$, and $k_{1} n$ and $\left(1-k_{1}\right) n$ are the number of edges from the middle column on the two sides of the graph partition, $0<k_{1}<1$. The term a $n\left(2 k_{1}-1\right)$ can be interpreted as the amount of imbalance between the denominators in the two terms in the $N$ cut formula and lies between -1 and +1 (since $0<a n<1)$. Simplifying, we see that

$$
n c u t^{*}=\frac{4 a n c}{c^{2}-\left(a n\left(2 k_{1}-1\right)\right)^{2}}<\frac{4 a n c}{c^{2}-1}=\frac{4 a n}{c-1 / c} .
$$

as was to be shown.

To complete the proof we must show that all other partitions result in a $N c u t$ greater than or equal to $\frac{4 a n}{c-1 / c}$. Informally speaking, what will happen is that either the numerators of the terms in the Ncut formula-the cut become too large, or the denominators become significantly imbalanced, again increasing the Ncut value. We need to consider three cases:

1. A cut that deviates from the cut in 1(b) slightly by reshuffling some of the $x_{i}$ edges so that the sums of the $x_{i}$ in each subset of the graph partition are no longer equal. For such cuts, the resulting Ncut values are, at best, $n c u t_{1}=\frac{2 a n}{c+x}+\frac{2 a n}{c-x}=\frac{4 a n c}{c^{2}-x^{2}}$. But, since $x \geq 1$, we have $n c u t_{1} \geq \frac{4 a n c}{c^{2}-1}=\frac{4 a n}{c-1 / c}$.

2. A cut that goes through any of the edges with weight $M$. Even with the denominators on both sides completely balanced, the Ncut value ncut $_{2}=\frac{2 M}{c}$ is going to be larger than $\frac{4 a n}{c-1 / c}$. This is ensured by our choice in the construction that $M>2 k^{2}$. We have to show that

$$
\begin{gathered}
\frac{2 M}{c} \geq \frac{4 a n}{c-1 / c}, \text { or } \\
M \geq 2 a n \frac{c^{2}}{c^{2}-1} .
\end{gathered}
$$

This is direct, since $a n<1$ by construction, $\frac{c^{2}}{c^{2}-1} \leq$ $\frac{81}{80}$ (using $k \geq 1, M \geq 2, c \geq 9$ ).

3. A cut that partitions out some of the nodes in the middle as one group. We see that any cut that goes through one of the $x_{i}$ s can improve its $N c u t$ value by going through the edges with weight $a$ instead. So, we will focus on the case where the cut only goes through the weight $a$ edges. Suppose that $m$ edges of $x_{i}$ s are grouped into one set, with total weight adding to $x$, where $1<x<2 k$. The corresponding ncut value,

$$
\begin{aligned}
\text { ncut }_{3}(m)= & \frac{4 a m}{4 a m+2 x} \\
& +\frac{4 a m}{8 M(n+1)+4 k+12 a n-4 a m-2 x} \\
= & \frac{2 a m}{c-d_{m}}+\frac{2 a m}{c+d_{m}},
\end{aligned}
$$

where 


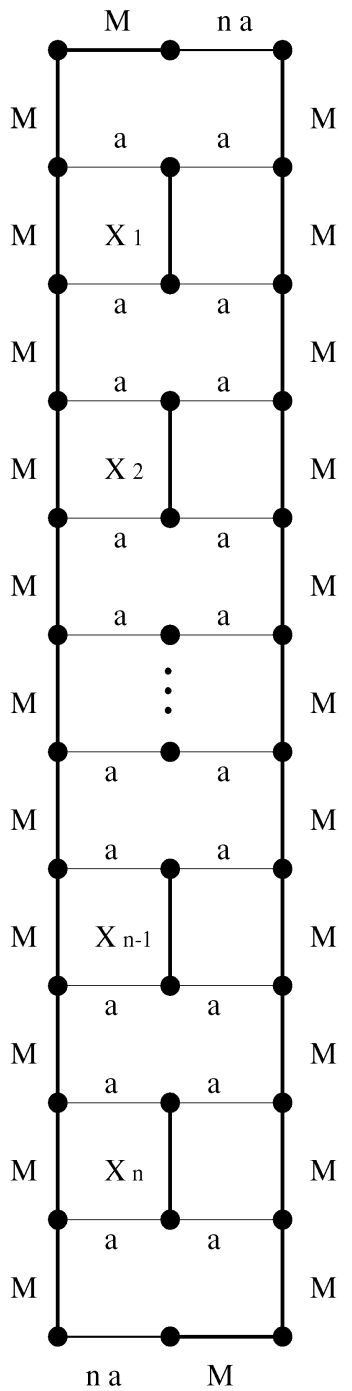

(a)

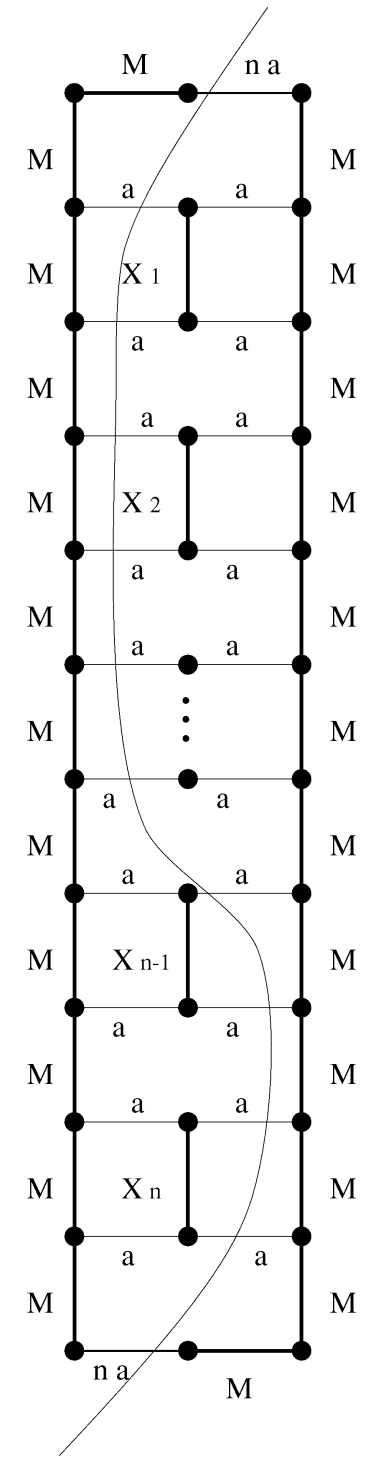

(b)
Fig. 18. (a) shows a weighted graph on a regular grid. The missing edges on the grids have weights of 0 . In comparison to the integers $x_{1}, x_{2}, \ldots, x_{n}, M$ is a large number $\left(M>2 k^{2}\right)$, and $a$ is very small number $(0<a<1 / n)$. (b) shows a cut that has a $N$ cut value less than $\frac{4 a n}{c-1 / c}$. This cut, which only goes through edges with weight equal to $a$ or $n a$, has the property that the $x_{i} \mathrm{~s}$ on each side of the partition sum up to $k$.

$$
\begin{aligned}
d_{m} & =2 M(n+1)+k+3 a n-2 a m-x \\
& >2 M(n+1)-k+3 a n-2 a m \\
& =x_{l} .
\end{aligned}
$$

The lower bound on $n_{c u t}(m)=\frac{4 a m c}{c^{2}-d_{m}^{2}}$ is then $n c u t_{l}(m)=\frac{4 a m c}{c^{2}-x_{l}^{2}}$. Further expansion of the $\operatorname{ncut}_{l}(m)$ yields

$$
\begin{aligned}
\text { ncut }_{l}(m)= & \frac{4 a m c}{c^{2}-x_{l}^{2}} \\
= & \frac{4 a m c}{c^{2}-(B-2 a m)^{2}}, \\
& \text { where } B=2 M(n+1)-k+3 a n \\
= & \frac{4 a c}{\frac{c^{2}-B^{2}}{m}-4 a^{2} m+4 a B} .
\end{aligned}
$$

One can check to see that $\operatorname{ncut}_{l}(m)$ is a nondecreasing function and has its minimum at $\frac{4 a c}{\left(c^{2}-B^{2}\right)+4 a B-4 a^{2}}$ when $m=1$.

In order to prove that $\operatorname{ncut}_{l}(m)>\frac{4 a n}{c-1 / c}$, we need to establish the inequality

$$
\begin{gathered}
\frac{4 a c}{\left(c^{2}-B^{2}\right)+4 a B-4 a^{2}} \geq \frac{4 a n c}{c^{2}-1} \text { or } \\
\frac{1}{\left(c^{2}-B^{2}\right)+4 a B-4 a^{2}} \geq \frac{n}{c^{2}-1} \text { or } \\
\left(\left(c^{2}-B^{2}\right)+4 a B-4 a^{2}\right) n \leq c^{2}-1 \text { or }
\end{gathered}
$$$$
\left(4 c k-4 k^{2}\right) n+4 a n(c-2 k-a)+1 \leq c^{2} \text {, }
$$

using the fact that $c=B+2 k$. To continue, note that, since an $<1$, this will be true if

$$
\begin{aligned}
\left(4 c k-4 k^{2}\right) n+4(c-2 k)-4 a+1 & \leq c^{2} \text { or if } \\
4 c k^{2}+4 c-\left(4 k^{3}+8 k+4 a-1\right) & \leq c^{2},
\end{aligned}
$$

since $n<k$. Since $4 k^{3}+8 k+4 a-1>0$, we only need to show that $4 c k^{2}+4 c<c^{2}$ or that $c>4\left(k^{2}+1\right)$. This is so because $c=2 M(n+1)+$ $k+3 a n$ and $M>2 k^{2}$.

\section{ACKNOWLEDGMENTS}

This research was supported by (ARO) DAAH04-96-1-0341, and a National Science Foundation Graduate Fellowship to J. Shi. We thank Christos Papadimitriou for supplying the proof of NP-completeness for normalized cuts on a grid. In addition, we wish to acknowledge Umesh Vazirani and Alistair Sinclair for discussions on graph theoretic algorithms and Inderjit Dhillon and Mark Adams for useful pointers to numerical packages. Thomas Leung, Serge Belongie, Yeir Weiss, and other members of the computer vision group at UC Berkeley provided much useful feedback on our algorithm.

\section{REFERENCES}

[1] N. Alon, "Eigenvalues and Expanders," Combinatorica, vol. 6, no. 2, pp. 83-96, 1986.

[2] A. Blake and A. Zisserman, Visual Reconstruction. MIT Press, 1987.

[3] R.B. Boppana, "Eigenvalues and Graph Bisection: An AverageCase Analysis," Proc. 28th Symp. Foundations of Computer Science, pp. 280-285, 1987.

[4] J. Cheeger, "A Lower Bound for the Smallest Eigenvalue of the Laplacian," Problems in Analysis, R.C. Gunning, ed., pp. 195-199, Princeton Univ. Press, 1970.

[5] F.R.K. Chung, Spectral Graph Theory. Am. Math. Soc., 1997.

[6] I.J. Cox, S.B. Rao, and Y. Zhong, "Ratio Regions: A Technique for Image Segmentation," Proc. 13th Int'l Conf. Pattern Recognition, 1996. 
[7] W.E. Donath and A.J. Hoffman, "Lower Bounds for the Partitioning of Graphs," IBM J. Research and Development, pp. 420-425, 1973.

[8] R. Van Driessche and D. Roose, "An Improved Spectral Bisection Algorithm and Its Application to Dynamic Load Balancing," Parallel Computing, vol. 21, pp. 29-48, 1995.

[9] M. Fiedler, "A Property of Eigenvectors of Nonnegative Symmetric Matrices and Its Applications to Graph Theory," Czech. Math. J., vol. 25, no. 100, pp. 619-633, 1975.

[10] S. Geman and D. Geman, "Stochastic Relaxation, Gibbs Distributions, and the Bayesian Restoration of Images," IEEE Trans. Pattern Analysis and Machine Intelligence, vol. 6, pp. 721-741, Nov. 1984.

[11] G.H. Golub and C.F. Van Loan, Matrix Computations. John Hopkins Press, 1989.

[12] A.K. Jain and R.C. Dubes, Algorithms for Clustering Data. Prentice Hall, 1988.

[13] K. Fukunaga, S. Yamada, H.S. Stone, and T. Kasai, "A Representation of Hypergraphs in the Euclidean Space," IEEE Trans. Computers, vol. 33, no. 4, pp. 364-367, Apr. 1984.

[14] Y.G. Leclerc, "Constructing Simple Stable Descriptions for Image Partitioning," Int'l J. Computer Vision, vol. 3, pp. 73-102, 1989.

[15] J. Malik, S. Belongie, J. Shi, and T. Leung, "Textons, Contours and Regions: Cue Integration in Image Segmentation," Proc. Int'l Conf. Computer Vision, pp. 918-925, 1999.

[16] J. Malik and P. Perona, "Preattentive Texture Discrimination with Early Vision Mechanisms," J. Optical Soc. Am., vol. 7, no. 2, pp. 923932, May 1990.

[17] D. Mumford and J. Shah, "Optimal Approximations by Piecewise Smooth Functions, and Associated Variational Problems," Comm. Pure Math., pp. 577-684, 1989.

[18] A. Pothen, H.D. Simon, and K.P. Liou, "Partitioning Sparse Matrices with Eigenvectors of Graphs," SIAM J. Matrix Analytical Applications, vol. 11, pp. 430-452, 1990.

[19] S. Sarkar and K.L. Boyer, "Quantitative Measures of Change Based on Feature Organization: Eigenvalues and Eigenvectors," Proc. IEEE Conf. Computer Vision and Pattern Recognition, 1996.

[20] J. Shi and J. Malik, "Normalized Cuts and Image Segmentation," Proc. IEEE Conf. Computer Vision and Pattern Recognition, pp. 731$737,1997$.

[21] J. Shi and J. Malik, "Motion Segmentation and Tracking Using Normalized Cuts," Proc. Int'l Conf. Computer Vision, pp. 1,1541,160, 1998.

[22] A.J. Sinclair and M.R. Jerrum, "Approximative Counting, Uniform Generation and Rapidly Mixing Markov Chains," Information and Computation, vol. 82, pp. 93-133, 1989.

[23] D.A. Spielman and S.H. Teng, "Disk Packings and Planar Separators," Proc. 12th ACM Symp. Computational Geometry, May 1996.

[24] M. Wertheimer, "Laws of Organization in Perceptual Forms (partial translation)," A Sourcebook of Gestalt Psycychology, W.B. Ellis, ed., pp. 71-88, Harcourt, Brace, 1938.

[25] Z. Wu and R. Leahy, "An Optimal Graph Theoretic Approach to Data Clustering: Theory and Its Application to Image Segmentation," IEEE Trans. Pattern Analysis and Machine Intelligence, vol. 15, no. 11, pp. 1,101-1,113, Nov. 1993.

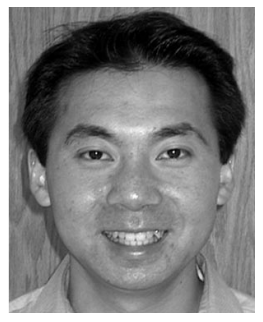

Jianbo Shi studied computer science and mathematics as an undergraduate at Cornell University where he recieved his BA degree in 1994. He received his $\mathrm{PhD}$ degree in computer science from the University of California at Berkeley in 1998. Since 1999, he has been a member of the faculty of the Robotics Institute at Carnegie Mellon University, where his primary research interests include image segmentation, grouping, object recognition, motion and shape analysis, and machine learning.

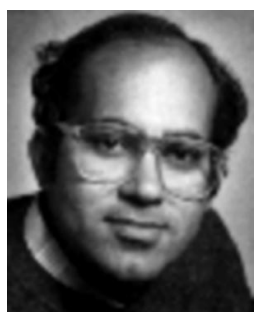

Jitendra Malik received the BTech degree in electrical engineering from the Indian Institute of Technology, Kanpur, in 1980 and the PhD degree in computer science from Stanford University in 1986. In January 1986, he joined the faculty of the Computer Science Division, Department of Electrical Engineering and Computer Science at the University of California at Berkeley, where he is currently a professor. During 1995-1998, he also served as vice-chair for graduate matters. He is a member of the Cognitive Science and Vision Science groups at UC Berkeley. His research interests are in computer vision and computational modeling of human vision. His work spans a range of topics in vision including image segmentation and grouping, texture, image-based modeling and rendering, content-based image querying, and intelligent vehicle highway systems. He has authored or coauthored more than 80 research papers on these topics.

$\mathrm{He}$ received the gold medal for the best graduating student in Electrical Engineering from IIT Kanpur in 1980, a Presidential Young Investigator Award in 1989, and the Rosenbaum fellowship for the Computer Vision Programme at the Newton Institute of Mathematical Sciences, University of Cambridge, in 1993. He is an editor-inchief of the International Journal of Computer Vision. He is a member of the IEEE. 